\title{
Effects of Nutritional Interventions on Cardiovascular Disease Health Outcomes in Aboriginal and Torres Strait Islander Australians: A Scoping Review
}

\author{
Bobby Porykali ${ }^{1,2,3, *,+}$, Alyse Davies $4,+\mathbb{D}$, Cassandra Brooks ${ }^{4,+}$, Hannah Melville ${ }^{4,+}$, \\ Margaret Allman-Farinelli ${ }^{4}$ (D) and Julieann Coombes ${ }^{3,5}$ (D)
}

1 School of Public Health, Faculty of Health, University of Technology Sydney, Sydney, NSW 2007, Australia

2 Indigenous Health Unit, Sydney Medical School, Faculty of Medicine and Health, University of Sydney, Sydney, NSW 2006, Australia

3 Aboriginal \& Torres Strait Islander Health Program, George Institute for Global Health, Newtown, NSW 2042, Australia; jcoombes@georgeinstitute.org.au

4 Charles Perkins Centre, Nutrition and Dietetics Group, Sydney School of Nursing, Faculty of Medicine and Health, University of Sydney, Sydney, NSW 2006, Australia; alyse.davies@sydney.edu.au (A.D.); cbro2650@uni.sydney.edu.au (C.B.); hmel2900@uni.sydney.edu.au (H.M.); margaret.allman-farinelli@sydney.edu.au (M.A.-F.)

5 Faculty of Medicine, University of New South Wales, Sydney, NSW 2052, Australia

* Correspondence: bobby.porykali@sydney.edu.au; Tel.: +61-02-8052-4495

+ These authors contributed equally to this work.

Citation: Porykali, B.; Davies, A.; Brooks, C.; Melville, H.;

Allman-Farinelli, M.; Coombes, J.

Effects of Nutritional Interventions on Cardiovascular Disease Health Outcomes in Aboriginal and Torres Strait Islander Australians: A Scoping Review. Nutrients 2021, 13, 4084.

https://doi.org/10.3390/nu13114084

Academic Editor: Ana Baylin

Received: 10 October 2021

Accepted: 12 November 2021

Published: 15 November 2021

Publisher's Note: MDPI stays neutral with regard to jurisdictional claims in published maps and institutional affiliations.

Copyright: (c) 2021 by the authors. Licensee MDPI, Basel, Switzerland. This article is an open access article distributed under the terms and conditions of the Creative Commons Attribution (CC BY) license (https:// creativecommons.org/licenses/by/ $4.0 /)$.

\begin{abstract}
Nutrition interventions can support Aboriginal and Torres Strait Islander peoples to reduce their risk of cardiovascular disease (CVD). This review examines nutritional interventions aiming to improve CVD outcomes and appraises peer-reviewed interventions using an Aboriginal and Torres Strait Islander Quality Appraisal Tool. Five electronic databases and grey literature were searched, applying no time limit. Two reviewers completed the screening, data extraction and quality assessment independently. The study quality was assessed using the South Australian Health and Medical Research Institute and the Centre of Research Excellence in Aboriginal Chronic Disease Knowledge Translation and Exchange Aboriginal and Torres Strait Islander Quality Appraisal Tool (QAT). Twenty-one nutrition programs were included in this review. Twelve reported on anthropometric measurements, ten on biochemical and/or hematological measurements and sixteen on other outcome domains. Most programs reported improvements in measurable CVD risk factors, including reduced body mass index (BMI), waist circumference (WC), weight, blood pressure and improved lipid profiles. Most programs performed well at community engagement and capacity strengthening, but many lacked the inclusion of Indigenous research paradigms, governance and strengths-based approaches. This review highlights the need for contemporary nutrition programs aimed at improving cardiovascular health outcomes to include additional key cultural components.
\end{abstract}

Keywords: Aboriginal; Torres Strait Islander; cardiovascular disease; first peoples; first nations; health promotion; nutrition

\section{Introduction}

Aboriginal and Torres Strait Islander Australians are strongly connected to "country" (land, waterways, and seas) through family, spiritual and traditional links that contribute to their health and wellbeing. History tells us that practices, policies and legislations directed towards Aboriginal and Torres Strait Islander peoples during Australia's early colonial years resulted in their forceful removal from lands and a disconnection from "country" that contributed to sudden changes in their dietary and lifestyle behaviours [1]. Traditionally, the nutrient composition of Aboriginal and Torres Strait Islander peoples' diet was high in protein, fibre, polyunsaturated fats and complex unrefined carbohydrates [2]. The low 
energy density of traditional diets along with the energy expended in food procurement processes provided a natural constraint on energy intake, and research suggests that precolonisation Aboriginal and Torres Strait Islander peoples were physically fit and lean [3]. The resulting loss of traditional lands, waterways and food practices, along with food insecurity and geographic factors, has changed their diet into a heavily processed western diet [4-6]. A western diet has been linked to the risk of developing Cardiovascular disease (CVD) and/or other complications as the diet features high amounts of saturated fat, red meat-based protein, added sugars, salt, processed and fast-foods, as well as low levels of fibre, certain micronutrients, wholegrains, fruit and vegetables [7].

Cardiovascular disease is a class of disorders affecting the heart and blood vessels and includes: coronary heart disease, ischemic heart disease, cerebrovascular disease, stroke, and others [8]. Cardiovascular disease risk factors include smoking, excessive alcohol, lack of physical activity, being overweight or obese, not eating the recommended amounts of fruit and vegetables and high blood pressure, blood glucose and cholesterol $[9,10]$. While CVD is a leading and serious disease in Australia [11], Aboriginal and Torres Strait Islander peoples have higher rates of CVD-related hospitalization and death compared to non-Indigenous Australians [12]. Addressing modifiable risk factors, such as diet, would help to prevent $37 \%$ of the burden of disease in Aboriginal and Torres Strait Islander populations [13].

The release of the Social Justice Report in 2005 resulted in a push towards achieving equality for Aboriginal and Torres Strait Islander peoples in relation to health and life expectancy [14]. Subsequently, this led to the collaborative initiation of Close the Gap campaign and the government response, through Closing the Gap [15]. One of the main considerations articulated for achieving equality in health outcomes is that research and programs are co-developed with communities and conducted in an appropriate way suitable to the needs, culture and paradigm of Aboriginal and Torres Strait Islander peoples. Further, there is an important emphasis on taking a strengths-based and preventative health approach $[16,17]$. Nutrition promotion is an important part of cardiovascularoriented health promotion and food and water security are key to allowing Aboriginal and Torres Strait Islander people to eat a healthy diet [18]. While previous health and nutrition programs have targeted Aboriginal and Torres Strait Islander peoples, none have been appropriately appraised to determine if they are culturally suitable, and few have been adequately evaluated $[19,20]$. Evaluations are crucial to understanding the viability, effectiveness and impact of nutrition programs as they inform nutrition policy and practice [21]. Moreover, they provide the evidence base necessary for funding and can enhance community support and awareness when released appropriately to stakeholders and the general public.

Cultural awareness, community engagement and partnerships, combined leadership, and sustainable resources are integral components of programs that aim to improve the health of Aboriginal and Torres Strait Islander peoples [19]. However, the existing frameworks and strategies used to guide the design and evaluation of Aboriginal and Torres Strait Islander nutritional programs have lacked appropriate tools to evaluate these programs and principles [22,23]. Aboriginal and Torres Strait Islander Quality Appraisal Tools (QAT) offers a credible and rigorous assessment to ensure programs are designed and delivered in a culturally appropriate manner [24]. A scoping review was selected for its ability to map all the relevant literature, including scientific peer-reviewed publications, as well as unpublished grey literature, to fill in the gaps in knowledge on this topic [25]. This review (1) examines the effects of nutrition interventions that aim to improve CVD outcomes in Aboriginal and Torres Strait Islander peoples; and (2) assesses the cultural appropriateness of programs with scientific peer-reviewed evaluations using the Aboriginal and Torres Strait Islander QAT. 


\section{Materials and Methods}

\subsection{Protocol and Registration}

The performance of this scoping review followed the Arksey \& O'Malley framework [26] and the findings are reported in accordance with the Joanna Briggs Institute updated methodological guidance for scoping reviews [27]. The protocol was developed and published on the Open Science Platform https: / / osf.io/z38gt/, accessed on 12 April 2021.

\subsection{Inclusion Criteria}

\subsubsection{Participants}

Aboriginal and Torres Strait Islander peoples of any age, with or without co-morbidities and residing in Australia were included. Programs were excluded if Aboriginal and Torres Strait Islander peoples did not make up more than $50 \%$ of the sample population or the data were unable to be extracted from non-indigenous Australians.

\subsubsection{Concept}

Programs or studies describing nutritional interventions that aim to improve CVD health outcomes were included. This included programs or studies with or without cointerventions targeting additional lifestyle diseases, as well as programs aiming to improve food security and dietary quality. Interventions that focused only on nutrient supplementation were excluded. For the published peer-reviewed studies, the interventions were required to have at least one marker from the following two outcome domains; (1) anthropometric measures (i.e., waist circumference (WC), body mass index (BMI), weight, hip measurements, waist-to-hip ratio (WHR) and fat mass); (2) biochemical and/or hematological biomarkers (i.e., total cholesterol (TC), high density lipoprotein cholesterol (HDL-C), low density lipoprotein cholesterol (LDL-C), triglycerides (TG), blood glucose level (BGL), systolic blood pressure (SBP), diastolic blood pressure (DBP), mean arterial blood pressure (MABP), fasting insulin and glycated haemoglobin (HbA1c). An additional program outcome category, "other", was included to capture any additional information (mostly relevant for non-published programs).

\subsubsection{Context}

This review considered programs conducted in the community at large from all the regions of Australia. This included programs delivered through outpatient facilities or clinics. Programs conducted in institutional settings (i.e., hospitals or aged care facilities) were excluded as disease or illnesses may influence dietary requirements and intake.

\subsection{Types of Studies}

To understand the extent, range and nature of all the programs aiming to improve CVD outcomes in Aboriginal and Torres Strait Islander peoples, the eligibility criteria for the included study types was broad. Primary study designs, conference proceedings, posters or abstracts, editorials, commentaries, perspectives, book chapters, newsletters, dissertations, theses, and government-produced reports and documents were all included. Systematic reviews and meta-analysis were excluded. The language of programs was restricted to English and there was no set date limit for the year of program dissemination.

\subsection{Search Strategy}

An initial limited search was conducted on MEDLINE and the Australian Indigenous HealthInfoNet to identify articles on the topic. A full search strategy was developed based on the key words contained in the titles and abstracts, as well as indexed terms used to classify the articles. A full search was conducted on Ovid (Medline and Embase), Scopus and Informit (Family and Society Collection and Indigenous Collection). Grey literature sources included the Australian Indigenous HealthInfoNet, Google Scholar and Lowitja Institute. The search strategy for MEDLINE is shown in Supplementary Table S1, 
conducted in March 2021. The reference lists of studies that were selected for full text screening were examined for additional programs.

\subsection{Selection Process}

The identified records from the full search were imported into EndNote X9.3.3. (Clarivate Analytics, PA, USA) for screening. The titles and abstracts of the retrieved studies were screened against the inclusion criteria by two independent reviewers (C.B. and H.M.). For the studies with the potential to be included, full texts were retrieved and attached in EndNote. Two reviewers screened against the inclusion and exclusion criteria independently. The reasons for exclusion after full text screening were recorded in EndNote and any discrepancies between the reviewers that arose throughout the process was resolved through discussion or consultation with a third reviewer (A.D.). The search results are presented in a PRISMA flow diagram (Figure 1).

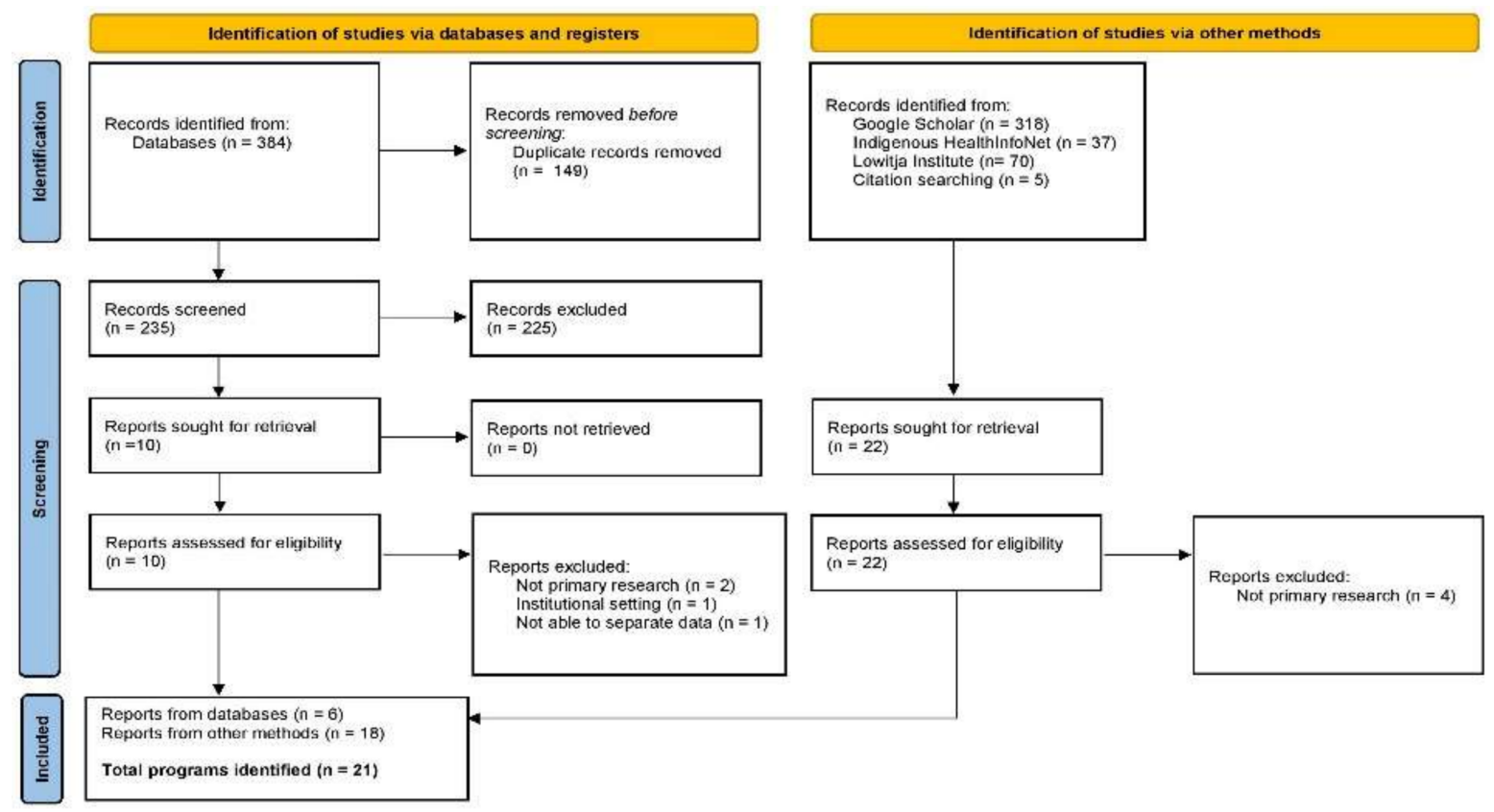

Figure 1. PRISMA Flow Diagram.

\subsection{Data Charting}

The data was charted using a standardized data charting form based on an existing framework for scoping reviews [26,27]. The following information was extracted for each included program: program reference, aim(s), intervention summary, timeframe (duration and time to follow up), target population, setting, outcome measures (anthropometric, biochemical and/or hematological biomarkers or other) and program status (completed, active or unclear). Using Microsoft Excel, two independent researchers (C.B. and H.M.) independently extracted data and resolved any discrepancies through discussion or consultation with a third reviewer (A.D.). In cases in which one program had multiple reports, all the reports were included if they met the inclusion criteria to ensure that the full details of the program were recorded. The authors of papers or relevant government bodies were contacted if missing or additional data needed to be requested.

\subsection{Critical Appraisal}

To assess the quality of the peer-reviewed research and its cultural appropriateness, the 2018 South Australian Health and Medical Research Institute (SAHMRI) and the Centre of Research Excellence in Aboriginal Chronic Disease Knowledge Translation and Exchange (CREATE) Aboriginal and Torres Strait Islander Quality Appraisal Tool (QAT) 
was implemented [24]. Two independent reviewers (C.B. and H.M.) used the Aboriginal and Torres Strait Islander Quality Appraisal Tool's Companion Document [28] as well as the Practical Guide for Researchers from the Lowitja Institute [29] to guide their response to the 14-question QAT, with disagreements resolved through consultation with a third Aboriginal reviewer (J.C.).

The responses to the QAT included yes, partial, unclear and no. The Yes category was assigned to studies that clearly provided written evidence in favour of the question. The Partial category was assigned to studies that provided some evidence, or brief evidence in favour of the question. The Unclear category was assigned to studies that may have actioned question items but did not explicitly report full or partial evidence. The No category was assigned to studies that did not report any evidence or reported evidence not in favour of the question item. These responses were presented in an adapted traffic light plot using the colours: green, blue, orange and red. A program was considered more culturally appropriate and of higher research quality if it received more "yes" responses. Inversely, a program was considered less culturally appropriate and of lower research quality if it received more "no" responses. A final rating of "very good" was assigned to papers that answered yes to over $75 \%$ of questions, "good" to those that answered yes to over $50 \%$ of questions, "fair" to those that answered yes to fewer than $50 \%$ of questions, and "poor" to those that answered yes to fewer than $25 \%$ of questions. To score how well each question performed, three points were assigned for "yes-green", two points for "partially-blue", one point for "unclear-orange" and no points for "no-red".

\subsection{Synthesis of Results}

The results are presented in tabular form, with an accompanying narrative summary to describe the results in relation to the scoping review's aims. Table 1 includes programs published in grey literature, while Table 2 includes programs published in scientific peerreviewed literature. The results were interpreted through a decolonized lens privileging Aboriginal and Torres Strait ways of knowing [30,31]. The findings of this review will be used to inform future community-led nutrition interventions aiming to improve CVD disease outcomes in Aboriginal and Torres Strait Islander peoples. 
Table 1. Program characteristics for unpublished literature $(n=12)$.

\begin{tabular}{|c|c|c|c|c|c|c|c|c|c|}
\hline $\begin{array}{l}\text { Program Name, } \\
\text { Reference, } \\
\text { Year }\end{array}$ & Aims & $\begin{array}{l}\text { Intervention } \\
\text { Summary }\end{array}$ & $\begin{array}{l}\text { Timeframe } \\
\text { (Duration/Time } \\
\text { to } \\
\text { Follow-up) }\end{array}$ & $\begin{array}{l}\text { Target } \\
\text { Population }\end{array}$ & Setting & $\begin{array}{l}\text { Anthropometric } \\
\text { Measurements }\end{array}$ & $\begin{array}{l}\text { Biochemical } \\
\text { and/or } \\
\text { Haematological } \\
\text { Biomarkers }\end{array}$ & $\begin{array}{l}\text { Other } \\
\text { Outcomes }\end{array}$ & Status \\
\hline $\begin{array}{l}\text { Living Strong } \\
\text { (formerly } \\
\text { known as the } \\
\text { Healthy Weight } \\
\text { Program) } \\
{[32]} \\
2005\end{array}$ & $\begin{array}{l}\text { To help } \\
\text { Aboriginal } \\
\text { and Torres } \\
\text { Strait Islander } \\
\text { peoples } \\
\text { maintain a } \\
\text { healthy } \\
\text { weight and } \\
\text { prevent } \\
\text { lifestyle } \\
\text { diseases }\end{array}$ & $\begin{array}{l}\text { Total participants }(n=432) \\
\text { Follow up }(n=34) \\
\text { QLD government-initiated weight } \\
\text { management and healthy lifestyle program } \\
\text { designed to teach lifestyle skills relating to } \\
\text { nutrition, PA and self-esteem } \\
\text { Delivery: In-person and group-based } \\
\text { Education: Healthy weight and weight loss; } \\
\text { behaviour change; low fat cooking; } \\
\text { budgeting for healthy meals; shopping tour; } \\
\text { self-esteem; benefits of exercise; } \\
\text { diabetes awareness }\end{array}$ & $\begin{array}{l}3 \text { months/ } \\
0,8,12 \mathrm{wks} \\
\text { (baseline, mid, } \\
\text { post program) }\end{array}$ & $\begin{array}{l}\text { Aboriginal and } \\
\text { Torres Strait } \\
\text { Islander adults }\end{array}$ & $\begin{array}{l}\text { QLD } \\
\text { (Program held } \\
\text { across } 8 \text { com- } \\
\text { munities; } \\
\text { Chermside, } \\
\text { Rockhamp- } \\
\text { ton, Mount } \\
\text { Morgan, } \\
\text { Blackwater, } \\
\text { Gehgre, } \\
\text { Woorabinda, } \\
\text { Toowoomba, } \\
\text { Wynnum) }\end{array}$ & $\begin{array}{l}\downarrow \text { Weight } \\
\text { (in }>50 \% \text { of } \\
\text { participants) } \\
\downarrow \text { WC } \\
\text { (in }>50 \% \text { of } \\
\text { participants) }\end{array}$ & N/A & $\begin{array}{l}\uparrow \text { Proportion of } \\
\text { participants eating } \\
\text { at least two daily } \\
\text { servings of fruit } \\
\uparrow \text { Proportion of } \\
\text { participants eating } \\
\text { five daily servings } \\
\text { of vegetables } \\
\uparrow \text { Reading NIP } \\
\uparrow \text { Water } \\
\uparrow \text { Using reduced } \\
\text { fat foods } \\
\uparrow \text { PA } \\
\text { (planned and } \\
\text { incidental) }\end{array}$ & Active \\
\hline $\begin{array}{l}\text { Life! Aboriginal } \\
\text { Road to Good } \\
\text { Health } \\
{[33]} \\
2016\end{array}$ & $\begin{array}{l}\text { To reduce the } \\
\text { risk of } \\
\text { developing } \\
\text { T2DM and } \\
\text { CVD in } \\
\text { Aboriginal } \\
\text { and Torres } \\
\text { Strait Islander } \\
\text { peoples }\end{array}$ & $\begin{array}{l}\text { Total participants } \\
\text { (unspecified) } \\
\text { Aboriginal and Torres Strait Islander healthy } \\
\text { lifestyle program, supported by the } \\
\text { Victorian government, within the "Life! } \\
\text { Program". The program is run by } \\
\text { Aboriginal health workers and Aboriginal } \\
\text { health services and included dietitians, } \\
\text { diabetes educators and personal trainers. } \\
\text { Culturally appropriate supporting resources } \\
\text { were used to assist in topic delivery } \\
\text { including facilitator manuals, participant } \\
\text { workbook material including recipes and } \\
\text { healthy eating and exercise books/posters } \\
\text { Delivery: six sessions delivered in-person as } \\
\text { a group course (telephone health coaching } \\
\text { service or the group course via zoom } \\
\text { available during the coronavirus pandemic) } \\
\text { Education: healthy eating; maintaining a } \\
\text { healthy weight; food label reading; } \\
\text { purchasing healthy/cost effective foods; } \\
\text { benefits of exercise; diabetes prevention; } \\
\text { cessation of smoking }\end{array}$ & $6 \mathrm{wks} / \mathrm{nil}$ & $\begin{array}{l}\text { Aboriginal and } \\
\text { Torres Strait } \\
\text { Islander } \\
\text { individuals, } \\
\text { families and } \\
\text { community } \\
\text { groups. } \\
\text { Eligibility } \\
>18 \text { and } \\
\text { diagnosed with } \\
\text { one or more of } \\
\text { the following: } \\
\text { (heart disease or } \\
\text { stroke, } \\
\text { gestational } \\
\text { diabetes, high } \\
\text { cholesterol, BP, } \\
\text { BGL, polycystic } \\
\text { ovarian } \\
\text { syndrome) }\end{array}$ & $\begin{array}{l}\text { VIC } \\
\text { (Melbourne) }\end{array}$ & N/A & N/A & $\begin{array}{l}\uparrow \text { Engagement } \\
\text { Feedback } \\
\text { (the flexible, fun, } \\
\text { family-friendly } \\
\text { approach in } \\
\text { addition to } \\
\text { a passionate } \\
\text { facilitator were } \\
\text { key factors to } \\
\text { its success) }\end{array}$ & Active \\
\hline
\end{tabular}


Table 1. Cont.

\begin{tabular}{|c|c|c|c|c|c|c|c|c|c|}
\hline $\begin{array}{l}\text { Program Name, } \\
\text { Reference, } \\
\text { Year }\end{array}$ & Aims & $\begin{array}{l}\text { Intervention } \\
\text { Summary }\end{array}$ & $\begin{array}{l}\text { Timeframe } \\
\text { (Duration/Time } \\
\text { to } \\
\text { Follow-up) }\end{array}$ & $\begin{array}{l}\text { Target } \\
\text { Population }\end{array}$ & Setting & $\begin{array}{l}\text { Anthropometric } \\
\text { Measurements }\end{array}$ & $\begin{array}{l}\text { Biochemical } \\
\text { and/or } \\
\text { Haematological } \\
\text { Biomarkers }\end{array}$ & $\begin{array}{l}\text { Other } \\
\text { Outcomes }\end{array}$ & Status \\
\hline $\begin{array}{l}\text { Pilbara } \\
\text { Aboriginal } \\
\text { Heart Health } \\
\text { Program } \\
{[34,35]} \\
2017 / 2018\end{array}$ & $\begin{array}{l}\text { To provide } \\
\text { comprehen- } \\
\text { sive, } \\
\text { coordinated, } \\
\text { integrated and } \\
\text { culturally } \\
\text { appropriate } \\
\text { health } \\
\text { education } \\
\text { services to the } \\
\text { Aboriginal } \\
\text { and Torres } \\
\text { Strait Islander } \\
\text { peoples }\end{array}$ & $\begin{array}{l}\text { Total participants } \\
\text { (unspecified) } \\
\text { Program developed in collaboration with } \\
\text { the Heart Foundation and Karratha Central } \\
\text { Healthcare. Incorporates culturally relevant } \\
\text { strategies including yarning to support } \\
\text { positive behaviour change. The program is } \\
\text { committed to the following principles: } \\
\text { community control, holistic approach, } \\
\text { cultural safety, equality, reciprocity and } \\
\text { inclusion, best practice, building community } \\
\text { capacity, accountability, sustainability. } \\
\text { Delivery: group-based, in-person activities } \\
\text { and meetings tailored to each community. } \\
\text { Education: healthy eating; heart health; } \\
\text { accessing health services; benefits of exercise }\end{array}$ & Unspecified & $\begin{array}{l}\text { Aboriginal and } \\
\text { Torres Strait } \\
\text { Islander peoples }\end{array}$ & $\begin{array}{l}\text { WA } \\
\text { (3 communi- } \\
\text { ties in the } \\
\text { Pilbara region: } \\
\text { Karratha, } \\
\text { Onslow and } \\
\text { Roebourne) }\end{array}$ & N/A & N/A & $\begin{array}{l}\uparrow \text { Self-esteem } \\
\uparrow \text { Knowledge } \\
\text { (healthy eating) } \\
\uparrow \mathrm{PA} \\
\uparrow \mathrm{CVD} \text { health } \\
\text { knowledge }\end{array}$ & Active \\
\hline $\begin{array}{l}\text { Healthy Eating } \\
\text { Activities and } \\
\text { Lifestyles for } \\
\text { Indigenous } \\
\text { Groups } \\
\text { (HEALInG) } \\
\text { [36] } \\
2007\end{array}$ & $\begin{array}{l}\text { To provide } \\
\text { realistic and } \\
\text { practical } \\
\text { information } \\
\text { on healthy } \\
\text { eating and } \\
\text { lifestyle } \\
\text { activities to } \\
\text { support } \\
\text { weight loss }\end{array}$ & $\begin{array}{l}\text { Total participants }(n=11) \\
\text { Goonellabah }(n=3) \\
\text { GurgunBulahnggelah } \\
(n=6 \text { to } 8) \\
\text { Program adapted from QLD Health's } \\
\text { Healthy Weight Program. } \\
\text { Delivery: in-person and group-based. One } \\
\text { hr exercise class, followed by an education } \\
\text { session on healthy eating and/or } \\
\text { lifestyle topics } \\
\text { Education: dietary guidelines; food groups; } \\
\text { serving sizes; reducing fat, salt and sugar } \\
\text { intake; food budgeting and label reading; } \\
\text { benefits of exercise. Information was also } \\
\text { provided on CVD, diabetes and } \\
\text { stroke prevention }\end{array}$ & $\begin{array}{l}10 \text { wks / } \\
0,10 \text { wks }\end{array}$ & $\begin{array}{l}\text { Aboriginal and } \\
\text { Torres Strait } \\
\text { Islander women }\end{array}$ & $\begin{array}{l}\text { NSW } \\
\text { (Northern } \\
\text { Rivers region } \\
\text { including } \\
\text { Goonellabah } \\
\text { and } \\
\text { GurgunBulah- } \\
\text { nggelah) }\end{array}$ & N/A & N/A & $\begin{array}{l}\uparrow \text { Knowledge and } \\
\text { skills relating to: } \\
\text { cooking healthy } \\
\text { and budget } \\
\text { conscious meals; } \\
\text { achieving daily PA } \\
\text { targets; food label } \\
\text { reading; fats, } \\
\text { sugar, and salt } \\
\text { content within } \\
\text { foods; chronic } \\
\text { diseases; and the } \\
\text { positive effects of } \\
\text { diet and exercise }\end{array}$ & Completed \\
\hline
\end{tabular}


Table 1. Cont.

\begin{tabular}{|c|c|c|c|c|c|c|c|c|c|}
\hline $\begin{array}{l}\text { Program Name, } \\
\text { Reference, } \\
\text { Year }\end{array}$ & Aims & $\begin{array}{l}\text { Intervention } \\
\text { Summary }\end{array}$ & $\begin{array}{l}\text { Timeframe } \\
\text { (Duration/Time } \\
\text { to } \\
\text { Follow-up) }\end{array}$ & $\begin{array}{l}\text { Target } \\
\text { Population }\end{array}$ & Setting & $\begin{array}{l}\text { Anthropometric } \\
\text { Measurements }\end{array}$ & $\begin{array}{l}\text { Biochemical } \\
\text { and/or } \\
\text { Haematological } \\
\text { Biomarkers }\end{array}$ & $\begin{array}{l}\text { Other } \\
\text { Outcomes }\end{array}$ & Status \\
\hline $\begin{array}{l}\text { Cooking } \\
\text { Healthy and } \\
\text { Physical } \\
\text { Activity } \\
\text { (CHAPA) } \\
\text { Project } \\
\text { [37] } \\
2009\end{array}$ & $\begin{array}{l}\text { To encourage } \\
\text { active self- } \\
\text { management } \\
\text { for people } \\
\text { with T2DM } \\
\text { and/or heart } \\
\text { disease }\end{array}$ & $\begin{array}{l}\text { Total participants (unspecified) } \\
\text { Group } 1(n=15) \\
\text { Program is one of four programs from } \\
\text { Healthy Active Australia "Community and } \\
\text { School Grants Program". Multidisciplinary } \\
\text { team including dietitians, exercise } \\
\text { physiologists and psychologists. } \\
\text { Delivery: in-person and group-based } \\
\text { Education: goal setting; healthy eating and } \\
\text { cooking; benefits PA }\end{array}$ & $\begin{array}{l}10 \mathrm{wks} / \mathrm{nil} \\
\text { (four groups, } \\
\text { over } 12 \text { months) }\end{array}$ & $\begin{array}{l}\text { People with heart } \\
\text { disease and/or } \\
\text { T2DM }\end{array}$ & $\begin{array}{l}\text { NT } \\
\text { (greater } \\
\text { Darwin } \\
\text { region) }\end{array}$ & N/A & N/A & $\begin{array}{l}\text { Anecdotal } \\
\text { remarks were } \\
\text { made on } \\
\text { improvements in } \\
\text { health, fitness, } \\
\text { weight, BP and } \\
\text { functional } \\
\text { strength }\end{array}$ & Completed \\
\hline $\begin{array}{l}\text { Koori Cook Off } \\
\text { Program } \\
{[38]} \\
2017\end{array}$ & $\begin{array}{l}\text { To improve } \\
\text { heart health } \\
\text { outcomes } \\
\text { through } \\
\text { nutrition } \\
\text { education } \\
\text { and } \\
\text { increase } \\
\text { confidence, } \\
\text { knowledge } \\
\text { and skills of } \\
\text { healthy cook- } \\
\text { ing } \\
\text { and eating }\end{array}$ & $\begin{array}{l}\text { Total participants (unspecified) } \\
\text { A cooking challenge program developed by } \\
\text { and for Aboriginal communities in } \\
\text { collaboration with the Heart Foundation. } \\
\text { Using healthy foods, groups cook healthy } \\
\text { meals for a panel of judges (local Elders) } \\
\text { Delivery: in-person and group-based, four } \\
\text { teams, each with four people } \\
\text { Education: culinary skills; creating healthy } \\
\text { meals using basic ingredients; portion sizes; } \\
\text { practical ways to increase FV consumption; } \\
\text { healthier oils; reducing salt; choosing } \\
\text { mainly water }\end{array}$ & nil/nil & $\begin{array}{l}\text { Aboriginal and } \\
\text { Torres Strait } \\
\text { Islander peoples }\end{array}$ & $\begin{array}{l}\text { NSW } \\
\text { (Communities } \\
\text { throughout } \\
\text { the Illawarra } \\
\text { and } \\
\text { Shoalhaven } \\
\text { regions) }\end{array}$ & N/A & N/A & $\begin{array}{l}\text { Anecdotal } \\
\text { feedback } \\
\text { suggested that the } \\
\text { program was } \\
\text { popular with the } \\
\text { community }\end{array}$ & Active \\
\hline
\end{tabular}


Table 1. Cont.

\begin{tabular}{|c|c|c|c|c|c|c|c|c|c|}
\hline $\begin{array}{l}\text { Program Name, } \\
\text { Reference, } \\
\text { Year }\end{array}$ & Aims & $\begin{array}{l}\text { Intervention } \\
\text { Summary }\end{array}$ & $\begin{array}{l}\text { Timeframe } \\
\text { (Duration/Time } \\
\text { to } \\
\text { Follow-up) }\end{array}$ & $\begin{array}{l}\text { Target } \\
\text { Population }\end{array}$ & Setting & $\begin{array}{l}\text { Anthropometric } \\
\text { Measurements }\end{array}$ & $\begin{array}{l}\text { Biochemical } \\
\text { and/or } \\
\text { Haematological } \\
\text { Biomarkers }\end{array}$ & $\begin{array}{l}\text { Other } \\
\text { Outcomes }\end{array}$ & Status \\
\hline $\begin{array}{l}\text { My Health for } \\
\text { Life (MH4L) } \\
{[39]} \\
2016\end{array}$ & $\begin{array}{l}\text { To decrease } \\
\text { participants' } \\
\text { risk of } \\
\text { developing } \\
\text { conditions } \\
\text { such as T2DM, } \\
\text { heart disease, } \\
\text { stroke, high } \\
\text { cholesterol } \\
\text { and high BP }\end{array}$ & $\begin{array}{l}\text { Total participants (unspecified) } \\
\text { Initiated by QLD government, now run by a } \\
\text { NGO partnership including Diabetes QLD, } \\
\text { National Heart Foundation, Stroke } \\
\text { Foundation and others. Participants must } \\
\text { complete a health check to participate. } \\
\text { A culturally appropriate tailored version of } \\
\text { the program was available for Aboriginal } \\
\text { and Torres Strait Islander peoples } \\
\text { Delivery: six-session program delivered } \\
\text { either remotely via telephone or } \\
\text { group-based and in-person. This followed } \\
\text { by a six-month online maintenance program } \\
\text { Education: healthy eating; benefits of } \\
\text { exercise; weight management; consuming } \\
\text { safe levels of alcohol; smoking cessation }\end{array}$ & 6 months/nil & $\begin{array}{l}\text { Aboriginal and } \\
\text { Torres Strait } \\
\text { Islander peoples } \\
\text { at risk of } \\
\text { developing } \\
\text { T2DM, heart } \\
\text { disease, stroke, } \\
\text { high cholesterol } \\
\text { and high BP }\end{array}$ & $\begin{array}{l}\text { QLD } \\
\text { (statewide) }\end{array}$ & $\mathrm{N} / \mathrm{A}$ & N/A & $\mathrm{N} / \mathrm{A}$ & Active \\
\hline $\begin{array}{l}\text { Gudbinji } \\
\text { Chronic Disease } \\
\text { Program } \\
\text { [40] } \\
\text { N/A }\end{array}$ & $\begin{array}{l}\text { To improve } \\
\text { heart health } \\
\text { and heart } \\
\text { health risk } \\
\text { factors }\end{array}$ & $\begin{array}{l}\text { Total participants (unspecified) } \\
\text { A one-day heart health focused program } \\
\text { that is part of a larger } 10 \mathrm{wk} \text { chronic disease } \\
\text { (including CVD) program } \\
\text { Delivery: In-person } \\
\text { Education: Healthy eating and cooking with } \\
\text { dietitians; benefits of exercise }\end{array}$ & 1 day/nil & $\begin{array}{l}\text { Aboriginal and } \\
\text { Torres Strait } \\
\text { Islander peoples }\end{array}$ & $\begin{array}{l}\text { NT } \\
\text { (Katherine, } \\
\text { Wurli- } \\
\text { Wurlinjang } \\
\text { Aboriginal } \\
\text { Health } \\
\text { Service) }\end{array}$ & $\mathrm{N} / \mathrm{A}$ & $\mathrm{N} / \mathrm{A}$ & $\mathrm{N} / \mathrm{A}$ & Active \\
\hline
\end{tabular}


Table 1. Cont.

\begin{tabular}{|c|c|c|c|c|c|c|c|c|c|}
\hline $\begin{array}{l}\text { Program Name, } \\
\text { Reference, } \\
\text { Year }\end{array}$ & Aims & $\begin{array}{l}\text { Intervention } \\
\text { Summary }\end{array}$ & $\begin{array}{l}\text { Timeframe } \\
\text { (Duration/Time } \\
\text { to } \\
\text { Follow-up) }\end{array}$ & $\begin{array}{l}\text { Target } \\
\text { Population }\end{array}$ & Setting & $\begin{array}{l}\text { Anthropometric } \\
\text { Measurements }\end{array}$ & $\begin{array}{l}\text { Biochemical } \\
\text { and/or } \\
\text { Haematological } \\
\text { Biomarkers }\end{array}$ & $\begin{array}{l}\text { Other } \\
\text { Outcomes }\end{array}$ & Status \\
\hline $\begin{array}{l}\text { Aboriginal and } \\
\text { Torres Strait } \\
\text { Islander Heart } \\
\text { Care Project } \\
\text { "Urimbirra } \\
\text { Geen" } \\
\text { [41] } \\
2000\end{array}$ & $\begin{array}{l}\text { To improve } \\
\text { heart disease } \\
\text { risk factors } \\
\text { and other } \\
\text { factors } \\
\text { affecting the } \\
\text { health of } \\
\text { Aboriginal } \\
\text { and Torres } \\
\text { Strait } \\
\text { Islanders }\end{array}$ & $\begin{array}{l}\text { Total participants (unspecified) } \\
\text { This multi-faceted project is a partnership } \\
\text { between government and NGOs addressing } \\
\text { heart health issues within the local } \\
\text { Aboriginal and Torres Strait } \\
\text { Islander community } \\
\text { Delivery: Through involvement with a } \\
\text { variety of different community initiatives } \\
\text { including: youth athletics carnival; } \\
\text { "Yandarra" - a healthy lifestyle partnership } \\
\text { project; Wagga Wagga Elders Physical } \\
\text { Activity Group; culturally appropriate } \\
\text { educational materials distributed by the } \\
\text { Greater Murray Area Health Service } \\
\text { Education: depend on the community } \\
\text { initiative involved but was centred around } \\
\text { healthy eating; packing healthy lunches and } \\
\text { snacks; PA; tobacco and alcohol }\end{array}$ & $\mathrm{N} / \mathrm{A}$ & $\begin{array}{l}\text { Aboriginal and } \\
\text { Torres Strait } \\
\text { Islander peoples }\end{array}$ & $\begin{array}{l}\text { NSW } \\
\text { (Wagga } \\
\text { Wagga) }\end{array}$ & $\mathrm{N} / \mathrm{A}$ & $\mathrm{N} / \mathrm{A}$ & $\mathrm{N} / \mathrm{A}$ & Unclear \\
\hline
\end{tabular}


Table 1. Cont.

\begin{tabular}{|c|c|c|c|c|c|c|c|c|c|}
\hline $\begin{array}{l}\text { Program Name, } \\
\text { Reference, } \\
\text { Year }\end{array}$ & Aims & $\begin{array}{l}\text { Intervention } \\
\text { Summary }\end{array}$ & $\begin{array}{l}\text { Timeframe } \\
\text { (Duration/Time } \\
\text { to } \\
\text { Follow-up) }\end{array}$ & $\begin{array}{l}\text { Target } \\
\text { Population }\end{array}$ & Setting & $\begin{array}{l}\text { Anthropometric } \\
\text { Measurements }\end{array}$ & $\begin{array}{l}\text { Biochemical } \\
\text { and/or } \\
\text { Haematological } \\
\text { Biomarkers }\end{array}$ & $\begin{array}{l}\text { Other } \\
\text { Outcomes }\end{array}$ & Status \\
\hline $\begin{array}{l}\text { Walkabout } \\
\text { Together } \\
{[43]} \\
2006\end{array}$ & $\begin{array}{l}\text { To address } \\
\text { high } \\
\text { community } \\
\text { levels of } \\
\text { chronic } \\
\text { disease } \\
\text { (including } \\
\text { hypertension } \\
\text { and diabetes) } \\
\text { via a nutrition } \\
\text { and PA } \\
\text { lifestyle } \\
\text { program }\end{array}$ & $\begin{array}{l}\text { Total participants }(n=150) \\
\text { Follow up }(n=126) \\
\text { Townsville Aboriginal and Islanders Health } \\
\text { Services developed this lifestyle } \\
\text { modification program to reduce impact of } \\
\text { chronic disease in the community } \\
\text { Delivery: in-person and group-based weekly } \\
\text { support sessions. Patients had access to GPs, } \\
\text { dietitians and health workers for regular } \\
\text { check-ups. Regular recreational walks } \\
\text { were encouraged } \\
\text { Education: unspecified }\end{array}$ & $1 \mathrm{yr} / 1 \mathrm{yr}$ & $\begin{array}{l}\text { Aboriginal and } \\
\text { Torres Strait } \\
\text { Islander peoples } \\
\text { (overweight; } \\
\text { BMI }>25 \mathrm{~kg} / \mathrm{m}^{2} \text { ) }\end{array}$ & $\begin{array}{l}\text { QLD } \\
\text { (Townsville } \\
\text { Aboriginal } \\
\text { and Islanders } \\
\text { Health } \\
\text { Service) }\end{array}$ & $\begin{array}{l}\downarrow \text { Weight } \\
\downarrow \text { WC }\end{array}$ & $\begin{array}{l}\downarrow \text { BGL } \\
\downarrow \text { TC } \\
\uparrow \text { HDL-C } \\
\downarrow \text { TG } \\
\downarrow \text { DBP } \\
\text { No change to } \\
\text { SBP and HbA1c }\end{array}$ & $\begin{array}{l}\text { Food/Nutrient } \\
\text { intake: } \uparrow \\
\text { Participants } \\
\text { consuming the } \\
\text { recommended } \\
\text { food group serves } \\
\text { from AGHE } \\
\text { PA: } \uparrow \text { Participants } \\
\text { performing } \\
\text { moderate and } \\
\text { vigorous PA >2 } \\
\text { two days/wk } \\
\uparrow \text { Steps } \\
\text { Correlation* } \\
\text { between daily } \\
\text { steps and } \\
\text { moderate and } \\
\text { vigorous PA } \\
\downarrow \text { Sedentary } \\
\text { behaviours } \\
\text { Other: } \\
\uparrow \text { Wellbeing * }\end{array}$ & Completed \\
\hline
\end{tabular}

\section{Coaching}

Patients on

Achieving

Cardiovascular

Health

$(\mathrm{COACH})$

Program

[44]
Total participants $(n=492)$ (included both Indigenous and non-Indigenous) distributed through the QLD Health Contact Centre,

this program is the first standardized

To reduce coaching program targeting CVD risk factors via telephone and mail outs Delivery: five coaching sessions by trained health professionals over 6 months, in form of telehealth (phone calls) and mail outs

Education: lifestyle modification; goal

setting; disease management

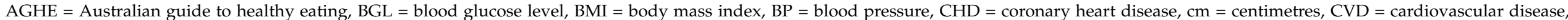

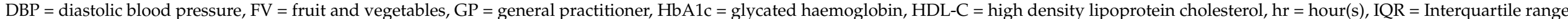

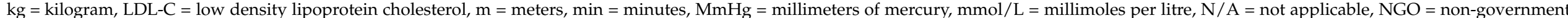

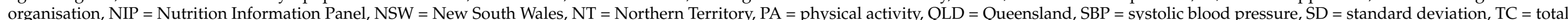

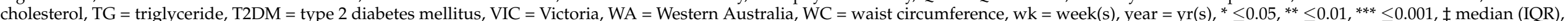
$\Delta$ change, $\downarrow$ decrease, $\uparrow$ increase. 
Table 2. Program characteristics for published literature $(n=9)$.

\begin{tabular}{|c|c|c|c|c|c|c|c|c|c|}
\hline $\begin{array}{l}\text { Program Name, } \\
\text { Reference, } \\
\text { Year }\end{array}$ & Aims & $\begin{array}{l}\text { Intervention } \\
\text { Summary }\end{array}$ & $\begin{array}{l}\text { Timeframe } \\
\text { (Duration/Time } \\
\text { to } \\
\text { Follow-up) }\end{array}$ & $\begin{array}{l}\text { Target } \\
\text { Population }\end{array}$ & Setting & $\begin{array}{l}\text { Anthropometric } \\
\text { Measurements }\end{array}$ & $\begin{array}{l}\text { Biochemical } \\
\text { and/or } \\
\text { Haematological } \\
\text { Biomarkers }\end{array}$ & $\begin{array}{l}\text { Other } \\
\text { Outcomes }\end{array}$ & Status \\
\hline $\begin{array}{l}\text { The Aboriginal } \\
\text { and Torres Strait } \\
\text { Islander } \\
\text { Women's } \\
\text { Fitness Program } \\
\text { [45] } \\
2012\end{array}$ & $\begin{array}{l}\text { To evaluate } \\
\text { the impact of } \\
\text { the program } \\
\text { on WC, } \\
\text { weight and } \\
\text { biomarkers } \\
\text { from baseline } \\
\text { (T1) to } \\
\text { immediately } \\
\text { post program } \\
\text { (T2) and to } \\
\text { assess if } \\
\text { outcomes } \\
\text { were } \\
\text { maintained at } \\
3 \text { month } \\
\text { follow-up (T3) }\end{array}$ & $\begin{array}{l}\text { Randomised Controlled Trial, Total } \\
\text { participants }(n=100) \\
\text { Significant lost to follow up and } \\
\text { missing data. } \\
\text { An exercise and nutrition program. The } \\
\text { cohort was split between an active group } \\
\text { and a waitlisted group (control) } \\
\text { Delivery: in-person and group-based. } \\
\text { Two one-hr cardiovascular and resistance } \\
\text { training classes per wk and four nutrition } \\
\text { education workshops } \\
\text { Education: food label reading; recipe } \\
\text { modification; cooking demonstration }\end{array}$ & $\begin{array}{l}12 \text { wks / } \\
12 \text { wks (T2) and } \\
3 \text { months (T3) }\end{array}$ & $\begin{array}{l}\text { Aboriginal and/or } \\
\text { Torres Strait } \\
\text { Islander women } \\
\text { aged } 18 \text { to } 64 \text { yrs } \\
\text { Participants must } \\
\text { have had a } \\
W C>80 \mathrm{~cm} \\
\text { Pregnant or } \\
\text { breastfeeding } \\
\text { (excluded) }\end{array}$ & $\begin{array}{l}\text { SA } \\
\text { (Adelaide) }\end{array}$ & $\begin{array}{l}\text { Association } \\
\text { between active } \\
\text { group and } \\
\text { control, } \\
\text { adjusting for all } \\
\text { potential } \\
\text { confounders } \\
\text { and T2DM } \\
\text { (T2) } \\
\downarrow \text { Weight } * \beta \\
\Delta 1.65 \mathrm{~kg} \\
\downarrow \mathrm{BMI} * \beta \\
\Delta 0.66 \mathrm{~kg} / \mathrm{m}^{2} \\
\downarrow \text { Waist and hip } \\
\text { measurements } \\
\text { (T3) } \\
\downarrow \text { Weight } * \beta \\
\Delta 2.50 \mathrm{~kg} \\
\downarrow \mathrm{BMI} * \beta \\
\Delta 1.03 \mathrm{~kg} / \mathrm{m}^{2} \\
\downarrow \text { Waist and hip } \\
\text { measurements }\end{array}$ & $\begin{array}{l}\text { Association between } \\
\text { active group and } \\
\text { control, adjusting for } \\
\text { all potential } \\
\text { confounders and } \\
\text { T2DM } \\
\text { (T2) } \\
\downarrow \text { SBP } \\
\downarrow \text { DBP } \\
\downarrow \text { HbA1c } \\
\downarrow \text { Glucose } \\
\downarrow \text { Insulin } \\
\text { TC (no change) } \\
\uparrow \text { TG } \\
\uparrow \text { LDL-C } \\
\downarrow \text { HDL-C } \\
\downarrow \text { CRP } \\
\text { (T3) } \\
\downarrow \text { SBP } \\
\downarrow \text { DBP } \\
\downarrow \text { HbA1c } \\
\downarrow \text { Glucose } \\
\uparrow \text { Insulin } \\
\downarrow \text { TC } \\
\downarrow \text { TG } \\
\uparrow \text { LDL-C } \\
\downarrow \text { HDL-C } \\
\downarrow \text { CRP }\end{array}$ & N/A & Completed \\
\hline
\end{tabular}


Table 2. Cont

\begin{tabular}{|c|c|c|c|c|c|c|c|c|c|}
\hline $\begin{array}{l}\text { Program Name, } \\
\text { Reference, } \\
\text { Year }\end{array}$ & Aims & $\begin{array}{l}\text { Intervention } \\
\text { Summary }\end{array}$ & $\begin{array}{l}\text { Timeframe } \\
\text { (Duration/Time } \\
\text { to } \\
\text { Follow-up) }\end{array}$ & $\begin{array}{l}\text { Target } \\
\text { Population }\end{array}$ & Setting & $\begin{array}{l}\text { Anthropometric } \\
\text { Measurements }\end{array}$ & $\begin{array}{l}\text { Biochemical } \\
\text { and/or } \\
\text { Haematological } \\
\text { Biomarkers }\end{array}$ & $\begin{array}{l}\text { Other } \\
\text { Outcomes }\end{array}$ & Status \\
\hline $\begin{array}{l}\text { The Healthy } \\
\text { Lifestyle } \\
\text { Programme } \\
\text { (HELP) } \\
{[46]} \\
2007\end{array}$ & $\begin{array}{l}\text { To determine } \\
\text { the } \\
\text { effectiveness } \\
\text { of lifestyle } \\
\text { intervention } \\
\text { on improving } \\
\text { diabetes and } \\
\text { cardiovascu- } \\
\text { lar risk } \\
\text { factors }\end{array}$ & $\begin{array}{l}\text { Cohort study, total participants }(n=101) \\
\text { Follow up }(n=80) \\
\text { A community-based, culturally } \\
\text { appropriate, lifestyle intervention to } \\
\text { improve cardiovascular risk factors. } \\
\text { Included: Self-monitoring of BGLs } \\
\text { and PA } \\
\text { Delivery: unspecified } \\
\text { Education: unspecified }\end{array}$ & $\begin{array}{l}2 \text { yrs } / 6 \text { months } \\
\text { (during } \\
\text { intervention) }\end{array}$ & $\begin{array}{l}\text { Aboriginal and } \\
\text { Torres Strait } \\
\text { Islanders, } \\
\text { overweight, } \\
>20 \text { yrs, } \\
\pm \text { T2DM }\end{array}$ & $\begin{array}{l}\text { QLD } \\
\text { (North } \\
\text { Stradbroke } \\
\text { Island and } \\
\text { Redland Bay) }\end{array}$ & $\begin{array}{l}\downarrow \mathrm{WC}^{* * \dagger}+ \\
\Delta 3.1 \mathrm{~cm} \\
\downarrow \mathrm{BMI} \\
\downarrow \mathrm{WHR}\end{array}$ & $\begin{array}{l}\downarrow \mathrm{DBP}^{* * \dagger} \\
\Delta 4.6 \mathrm{mmHg} \\
\downarrow \mathrm{SBP} \\
\downarrow \mathrm{MABP} * \dagger \\
\Delta 4.2 \mathrm{mmHg} \\
\downarrow \mathrm{TC}^{* * \dagger} \\
\Delta 0.26 \mathrm{mmol} / \mathrm{L} \\
\downarrow \mathrm{TG}^{* \dagger} \\
\Delta 0.18 \mathrm{mmol} / \mathrm{L} \\
\downarrow \mathrm{HDL}-\mathrm{C}^{* * * \dagger} \\
\Delta 0.09 \mathrm{mmol} / \mathrm{L} \\
\downarrow \mathrm{LDL}-\mathrm{C} \\
\uparrow \mathrm{Hb} 1 \mathrm{c}^{* * *} \dagger \\
\Delta 0.31 \% \\
\uparrow \text { Fasting BGL }\end{array}$ & $\uparrow$ Steps & Completed \\
\hline $\begin{array}{l}\text { Cardiopulmonary } \\
\text { rehabilitation } \\
\text { and secondary } \\
\text { prevention } \\
\text { program } \\
{[47]} \\
2014\end{array}$ & $\begin{array}{l}\text { To improve } \\
\text { health } \\
\text { outcomes of } \\
\text { Aboriginal } \\
\text { and } \\
\text { Torres Strait } \\
\text { Islanders with } \\
\text { diagnosed } \\
\text { CVD and/or } \\
\text { associated risk } \\
\text { factors }\end{array}$ & $\begin{array}{l}\text { Cohort study, total participants }(n=92) \\
\text { Follow up }(n=72) \\
\text { Cardio-pulmonary programs }(n=13) \\
\text { developed and delivered under an } \\
\text { Aboriginal community-controlled health } \\
\text { service. It had two components: } \\
\text { education and exercise } \\
\text { Delivery: in-person and group-based. } \\
\text { Two one-hr exercises and one one-hr } \\
\text { education session per wk } \\
\text { Education: CVD; benefits of exercise; } \\
\text { shopping; cooking and eating healthy } \\
\text { food; medication usage; risks of smoking; } \\
\text { stress reduction techniques }\end{array}$ & $\begin{array}{l}8 \text { wks/ } \\
8 \text { wks }\end{array}$ & $\begin{array}{l}\text { Aboriginal and/or } \\
\text { Torres Strait } \\
\text { Islanders with a } \\
\text { diagnosis of } \\
\text { COPD, IHD or } \\
\text { CHF, and at least } \\
\text { two } \\
\text { cardiovascular } \\
\text { risk factors } \\
\text { (smoking, obesity, } \\
\text { hypertension, } \\
\text { diabetes, } \\
\text { dyslipidemia) }\end{array}$ & $\begin{array}{l}\text { TAS } \\
\text { (Launceston } \\
\text { and Hobart) }\end{array}$ & $\begin{array}{l}\text { Participants } \\
\text { with risk factors } \\
\downarrow \text { Weight } \\
\Delta 0.8 \mathrm{~kg} \\
(\mathrm{ES}=0.04) \\
\downarrow \mathrm{BMI} \\
\Delta 0.3 \mathrm{~kg} / \mathrm{m}^{2} \\
(\mathrm{ES}=0.03) \\
\downarrow \mathrm{WC} \Delta 3.0 \mathrm{~cm} \\
(\mathrm{ES}=0.17)\end{array}$ & N/A & $\begin{array}{l}\text { Participants } \\
\text { with risk } \\
\text { factors } \\
\uparrow \text { Six-min } \\
\text { walk } \\
\text { distance } † \\
\Delta 43.6 \mathrm{~m} \\
(\mathrm{ES}=0.10) \\
\downarrow \text { Dyspnoea } \\
\downarrow \text { Fatigue } \\
\uparrow \text { Quality } \\
\text { of life }\end{array}$ & Completed \\
\hline
\end{tabular}


Table 2. Cont

\begin{tabular}{|c|c|c|c|c|c|c|c|c|c|}
\hline $\begin{array}{l}\text { Program Name, } \\
\text { Reference, } \\
\text { Year }\end{array}$ & Aims & $\begin{array}{l}\text { Intervention } \\
\text { Summary }\end{array}$ & $\begin{array}{l}\text { Timeframe } \\
\text { (Duration/Time } \\
\text { to } \\
\text { Follow-up) }\end{array}$ & $\begin{array}{l}\text { Target } \\
\text { Population }\end{array}$ & Setting & $\begin{array}{l}\text { Anthropometric } \\
\text { Measurements }\end{array}$ & $\begin{array}{l}\text { Biochemical } \\
\text { and/or } \\
\text { Haematological } \\
\text { Biomarkers }\end{array}$ & $\begin{array}{l}\text { Other } \\
\text { Outcomes }\end{array}$ & Status \\
\hline $\begin{array}{l}\text { Heart } \\
\text { Health-For } \\
\text { Our People, by } \\
\text { Our People } \\
{[48,49]} \\
2013 / 2012\end{array}$ & $\begin{array}{l}\text { To evaluate } \\
\text { the uptake } \\
\text { and effects on } \\
\text { lifestyle, and } \\
\text { cardiovascu- } \\
\text { lar risk factors, } \\
\text { of cardiac } \\
\text { rehabilitation } \\
\text { at an AMS }\end{array}$ & $\begin{array}{l}\text { Cross sectional study, total participants } \\
(n=120), 18 \text { months since } \\
\text { program commencement } \\
\text { A culturally appropriate cardiac } \\
\text { rehabilitation program focused on } \\
\text { improving cardiovascular health through } \\
\text { nutrition, PA and lifestyle } \\
\text { Delivery: in-person and group-based. } \\
\text { Weekly education sessions utilising } \\
\text { yarning and PA } \\
\text { Education: healthy eating; heart health } \\
\text { risk factor modification; diabetes; } \\
\text { management of medications; healthy } \\
\text { tucker; healthy weight; oral health; stress } \\
\text { and emotion management; PA }\end{array}$ & $\begin{array}{l}\text { Unspecified/ } \\
8 \text { wk snapshot } \\
\text { data of } \\
28 \text { participants } \\
\text { (20 female) }\end{array}$ & $\begin{array}{l}\text { Aboriginal and } \\
\text { Torres Strait } \\
\text { Islander peoples } \\
\text { with or at risk of } \\
\text { chronic disease }\end{array}$ & $\begin{array}{l}\text { WA } \\
\text { (Derbarl } \\
\text { Yerrigan } \\
\text { Health } \\
\text { Service, Perth) }\end{array}$ & $\begin{array}{l}\downarrow \text { BMI * } \\
\Delta 34.0 \mathrm{~kg} / \mathrm{m}^{2} \\
(5.1) \text { to } \\
33.3 \mathrm{~kg} / \mathrm{m}^{2}(5.2) \\
\downarrow W C^{* *+} \\
\Delta 113 \mathrm{~cm}(14) \text { to } \\
109 \mathrm{~cm}(13) \\
\downarrow \text { Weight }\end{array}$ & $\begin{array}{l}\downarrow \mathrm{SBP}^{* \dagger} \\
\Delta 135 \mathrm{mmHg}(20) \text { to } \\
120 \mathrm{mmHg}(16) \\
\downarrow \mathrm{DBP}^{* \dagger} \\
\Delta 78 \mathrm{mmHg}(12) \text { to } \\
72 \mathrm{mmHg}(5)\end{array}$ & $\begin{array}{l}\uparrow 6 \text { min walk } \\
\text { distance } \\
\Delta 296 \mathrm{~m} \mathrm{(115)} \\
\text { to } 345 \mathrm{~m} \mathrm{(135)}\end{array}$ & Active \\
\hline $\begin{array}{l}\text { Gut } \\
\text { Busters } \\
{[50]} \\
1999\end{array}$ & $\begin{array}{l}\text { To promote } \\
\text { long-term } \\
\text { lifestyle } \\
\text { changes and } \\
\text { enable } \\
\text { community } \\
\text { ownership } \\
\text { and } \\
\text { continuation }\end{array}$ & $\begin{array}{l}\text { Cohort study, total participants }(n=57) \\
\text { Follow-up }(n=47) \\
\text { An NSW Health program developed in } \\
\text { 1991, adapted for Aboriginal and Torres } \\
\text { Strait Islander men. A "waist loss" } \\
\text { program that made sustainable lifestyle } \\
\text { changes across the community through } \\
\text { the recruitment of Indigenous } \\
\text { male leaders } \\
\text { Delivery: in-person and group-based } \\
\text { Education: reducing fat intake; increasing } \\
\text { dietary fibre; increasing daily movement; } \\
\text { changing 'obesogenic' habits }\end{array}$ & $\begin{array}{l}12 \text { months } / 2 \text {, } \\
6 \text { and } 12 \text { months } \\
\text { (during } \\
\text { intervention) }\end{array}$ & $\begin{array}{l}\text { Aboriginal and/or } \\
\text { Torres Strait } \\
\text { Islander men }\end{array}$ & $\begin{array}{l}\text { NT } \\
\text { (4 island } \\
\text { groups in the } \\
\text { Torres Strait } \\
\text { region of } \\
\text { Northern } \\
\text { Australia) }\end{array}$ & $\begin{array}{l}\downarrow \text { Weight } * * *+ \\
\Delta 107 \mathrm{~kg}(18.2) \\
\text { to } 103 \mathrm{~kg}(18.1) \\
\downarrow \text { WC } * * *+ \\
\Delta 118 \mathrm{~cm}(13.6) \\
\text { to } 114 \mathrm{~cm}(13.9) \\
\downarrow \text { BMI ***t } \\
\Delta 34.7 \mathrm{~kg} / \mathrm{m}^{2} \\
(5.4) \text { to } \\
33.6 \mathrm{~kg} / \mathrm{m}^{2}(5.4) \\
\downarrow \text { WHR } * * * \dagger \\
\Delta 1.05(0.05) \text { to } \\
0.98(0.05) \\
\downarrow \text { Fat mass } * * * \dagger \\
\Delta 36.7 \mathrm{~kg}(12.2) \\
\text { to } 32.8 \mathrm{~kg}(12.5) \\
\downarrow \text { Body Fat }(\%) \\
\Delta 34(4.9) \text { to } \\
32(5.6) * * *\end{array}$ & N/A & N/A & $\begin{array}{l}\text { Unclear } \\
\text { (may still be } \\
\text { active in the } \\
\text { community) }\end{array}$ \\
\hline
\end{tabular}


Table 2. Cont

\begin{tabular}{|c|c|c|c|c|c|c|c|c|c|}
\hline $\begin{array}{l}\text { Program Name, } \\
\text { Reference, } \\
\text { Year }\end{array}$ & Aims & $\begin{array}{l}\text { Intervention } \\
\text { Summary }\end{array}$ & $\begin{array}{l}\text { Timeframe } \\
\text { (Duration/Time } \\
\text { to } \\
\text { Follow-up) }\end{array}$ & $\begin{array}{l}\text { Target } \\
\text { Population }\end{array}$ & Setting & $\begin{array}{l}\text { Anthropometric } \\
\text { Measurements }\end{array}$ & $\begin{array}{l}\text { Biochemical } \\
\text { and/or } \\
\text { Haematological } \\
\text { Biomarkers }\end{array}$ & $\begin{array}{l}\text { Other } \\
\text { Outcomes }\end{array}$ & Status \\
\hline $\begin{array}{l}\text { Unity of First } \\
\text { People of } \\
\text { Australia } \\
\text { Diabetes } \\
\text { Management } \\
\text { Care Program } \\
\text { (UFPA) } \\
\text { [51] } \\
2006\end{array}$ & $\begin{array}{l}\text { To prevent } \\
\text { chronic } \\
\text { diseases, } \\
\text { including } \\
\text { CVD, T2DM } \\
\text { and obesity }\end{array}$ & $\begin{array}{l}\text { Cohort Study, total participants } \\
\text { (unspecified) Population of sum for the } \\
\text { four communities ( } n=1350 \text { ) } \\
\text { An Aboriginal-run screening and } \\
\text { intervention program designed to } \\
\text { increase awareness of chronic disease; } \\
\text { promote healthier living; increase } \\
\text { screening; advocate for early treatment; } \\
\text { increase medication compliance; reduce } \\
\text { further health complications } \\
\text { Delivery: community-based and } \\
\text { in-person. Group and individual } \\
\text { level intervention. } \\
\text { Education: diabetes education; PA; diet } \\
\text { and nutrition; lifestyle; self-management; } \\
\text { weight reduction; health education }\end{array}$ & $\begin{array}{l}\text { Unspecified, } \\
\text { "many months } \\
\text { to } 3 \text { yrs in the } \\
\text { different } \\
\text { communi- } \\
\text { ties"/Unspecified }\end{array}$ & $\begin{array}{l}\text { Aboriginal and } \\
\text { Torres Strait } \\
\text { Islanders of all } \\
\text { ages }\end{array}$ & $\begin{array}{l}\text { WA } \\
\text { (4 communi- } \\
\text { ties Gibson } \\
\text { Desert in } \\
\text { Pilbara, } \\
\text { Fitzroy Valley } \\
\text { West } \\
\text { Kimberley, } \\
\text { and two } \\
\text { communities } \\
\text { inEast } \\
\text { Kimberley) }\end{array}$ & $\begin{array}{l}\text { Findings } \\
\text { reported for one } \\
\text { Kimberly } \\
\text { community } \\
\text { (better findings } \\
\text { reported for } \\
\text { diabetic rather } \\
\text { than } \\
\text { non-diabetic } \\
\text { persons): } \\
\downarrow \text { Weight } \\
\downarrow \text { BMI } \\
\downarrow \text { WC }\end{array}$ & $\begin{array}{l}\text { Findings reported } \\
\text { for one Kimberly } \\
\text { community: } \\
\downarrow \text { HbA1c } \\
\downarrow \text { TC } \\
\downarrow \text { LDL-C } \\
\uparrow \text { HDL-C }\end{array}$ & $\uparrow \mathrm{PA}$ & Completed \\
\hline
\end{tabular}


Table 2. Cont

\begin{tabular}{|c|c|c|c|c|c|c|c|c|c|}
\hline $\begin{array}{l}\text { Program Name, } \\
\text { Reference, } \\
\text { Year }\end{array}$ & Aims & $\begin{array}{l}\text { Intervention } \\
\text { Summary }\end{array}$ & $\begin{array}{l}\text { Timeframe } \\
\text { (Duration/Time } \\
\text { to } \\
\text { Follow-up) }\end{array}$ & $\begin{array}{l}\text { Target } \\
\text { Population }\end{array}$ & Setting & $\begin{array}{l}\text { Anthropometric } \\
\text { Measurements }\end{array}$ & $\begin{array}{l}\text { Biochemical } \\
\text { and/or } \\
\text { Haematological } \\
\text { Biomarkers }\end{array}$ & $\begin{array}{l}\text { Other } \\
\text { Outcomes }\end{array}$ & Status \\
\hline $\begin{array}{l}\text { The Minjilang } \\
\text { Health and } \\
\text { Nutrition } \\
\text { Project } \\
\text { [52] } \\
1994\end{array}$ & $\begin{array}{l}\text { To measure } \\
\text { nutritional } \\
\text { status of } \\
\text { adults at } \\
\text { Minjilang and } \\
\text { describe } \\
\text { community } \\
\text { dietary intake, } \\
\text { and use data } \\
\text { for planning, } \\
\text { implement- } \\
\text { ing, and } \\
\text { monitoring } \\
\text { and } \\
\text { evaluation of } \\
\text { intervention }\end{array}$ & $\begin{array}{l}\text { Cohort study, total participants } \\
\text { Minjilang }(n=154) \\
\text { Control community }(n=310) \\
\text { (during intervention period) } \\
\text { Program included health screening } \\
\text { (voluntary), intervention and evaluation } \\
\text { against a comparison community } \\
\text { Delivery: community-delivered via } \\
\text { Minjilang Clinic } \\
\text { Education: encouraged FV intake and } \\
\text { lean meats similar to traditional } \\
\text { bush foods; } \\
\text { discourage T/A and sugary foods; } \\
\text { exercise was encouraged }\end{array}$ & $\begin{array}{l}12 \text { months } / 3,6 \text {, } \\
9,12 \text { (during } \\
\text { intervention) }\end{array}$ & $\begin{array}{l}\text { Aboriginal and } \\
\text { Torres Strait } \\
\text { Islanders adults }\end{array}$ & $\begin{array}{l}\text { NT } \\
\text { (Minjilang, } \\
\text { Croker Island) }\end{array}$ & $\downarrow \mathrm{BMI} * * *$ & $\begin{array}{l}\downarrow \mathrm{DBP} * * * \\
\downarrow \mathrm{SBP} * * * \\
\downarrow \mathrm{TC} * * *+ \\
\Delta 12.3 \% \\
\downarrow \text { Fasting TG } \\
\text { (nondiabetics only) } \\
\uparrow \mathrm{RBC} \\
\uparrow \text { Serum folate } \\
\uparrow \text { Serum } \mathrm{B}_{6} \\
\uparrow \text { Plasma B-carotene } \\
\uparrow \text { Plasma ascorbic } \\
\text { acid }\end{array}$ & $\begin{array}{l}\uparrow \text { FV } \\
\downarrow \text { Sugar } \\
\uparrow \text { low sugar } \\
\text { drinks } \\
\downarrow \text { T/A food } \\
\uparrow \text { Wholemeal } \\
\text { bread } \\
\downarrow \% \text { E from } \\
\text { total and } \\
\text { saturated fat } \\
\text { MUFA and } \\
\text { PUFA oils } \\
\text { replaced other } \\
\text { oils } \\
\downarrow \% \text { from } \\
\text { sugars } \\
\uparrow \text { Dietary } \\
\text { density of } \\
\text { ascorbic acid, } \\
\text { b-carotene, } \\
\text { thiamine, } \\
\text { folate, calcium } \\
\uparrow \text { Fibre }\end{array}$ & $\begin{array}{l}\text { Unclear } \\
\text { (may still be } \\
\text { active in the } \\
\text { community) }\end{array}$ \\
\hline $\begin{array}{l}\text { Unnamed } \\
{[53]} \\
2000\end{array}$ & $\begin{array}{l}\text { To raise } \\
\text { community } \\
\text { awareness of } \\
\text { diabetes and } \\
\text { CVD }\end{array}$ & $\begin{array}{l}\text { Total participants }(1987 n=348,1991 \\
n=331,1995 n=305) \\
\text { Community-based nutrition awareness } \\
\text { healthy lifestyle program } \\
\text { Delivery: unspecified } \\
\text { Education: diabetes awareness; } \\
\text { healthy food-buying }\end{array}$ & $2 \mathrm{yrs} / 3,8 \mathrm{yrs}$ & $\begin{array}{l}\text { Aboriginal } \\
\text { community } \\
\text { members > } 15 \text { yrs }\end{array}$ & $\begin{array}{l}\text { Central } \\
\text { Australia }\end{array}$ & $\begin{array}{l}\text { At } 8 \text { yr } \\
\text { follow up } \\
\uparrow \text { Obesity } \\
\text { (OR: } 1.84 \text { ) } \\
\text { Women } \\
\text { (change } \\
\text { over time) } \\
\uparrow \text { BMI ***† } \\
\uparrow \mathrm{WC}^{* * *+}\end{array}$ & 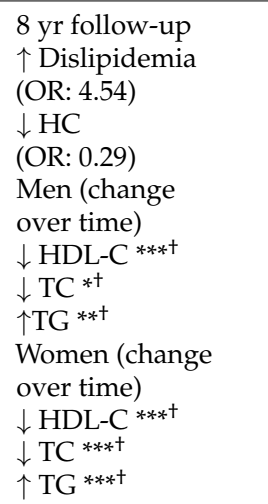 & $\begin{array}{l}\text { Food/Nutrient } \\
\text { intake: } \\
\downarrow \% \text { E from fat } \\
\text { and saturated } \\
\text { fat } \\
\downarrow \% \text { E from } \\
\text { sugar } \\
\uparrow \text { Complex } \\
\text { CHO intake } \\
\text { Store turnover } \\
\text { method: } \\
\downarrow \text { FV } \\
\downarrow \text { Sugar } \\
\uparrow \text { Flour and } \\
\text { bread }\end{array}$ & Completed \\
\hline
\end{tabular}


Table 2. Cont

\begin{tabular}{|c|c|c|c|c|c|c|c|c|c|}
\hline $\begin{array}{l}\text { Program Name, } \\
\text { Reference, } \\
\text { Year }\end{array}$ & Aims & $\begin{array}{l}\text { Intervention } \\
\text { Summary }\end{array}$ & $\begin{array}{l}\text { Timeframe } \\
\text { (Duration/Time } \\
\text { to } \\
\text { Follow-up) }\end{array}$ & $\begin{array}{l}\text { Target } \\
\text { Population }\end{array}$ & Setting & $\begin{array}{l}\text { Anthropometric } \\
\text { Measurements }\end{array}$ & $\begin{array}{l}\text { Biochemical } \\
\text { and/or } \\
\text { Haematological } \\
\text { Biomarkers }\end{array}$ & $\begin{array}{l}\text { Other } \\
\text { Outcomes }\end{array}$ & Status \\
\hline $\begin{array}{l}\text { The Looma } \\
\text { Healthy } \\
\text { Lifestyle Project } \\
{[54,55]} \\
2000 / 2001\end{array}$ & $\begin{array}{l}\text { To reduce } \\
\text { CHD through } \\
\text { dietary } \\
\text { modification }\end{array}$ & $\begin{array}{l}\text { Cross-sectional study, total participants } \\
(n=49) \\
\text { (32 intervention, } 17 \text { control) } \\
\text { Cross-sectional community samples } \\
\text { (baseline } n=200 \text {, two-yrs } n=185, \\
\text { four yrs } n=132) \\
\text { Delivery: in-person and group-based. } \\
\text { Education: healthy cooking techniques; } \\
\text { sources of refined CHO; importance of } \\
\text { FV; store tours; benefits of exercise }\end{array}$ & $\begin{array}{l}2 \text { yrs/ } \\
2,6,12,18, \\
24 \text { months } \\
\text { (during } \\
\text { intervention) }\end{array}$ & $\begin{array}{l}\text { Aboriginal and } \\
\text { Torres Strait } \\
\text { Islanders at high } \\
\text { risk of developing } \\
\text { diabetes and CHD }\end{array}$ & $\begin{array}{l}\text { WA } \\
\text { (Looma } \\
\text { Aboriginal } \\
\text { community, in } \\
\text { the remote } \\
\text { Kimberley } \\
\text { region) }\end{array}$ & $\begin{array}{l}\downarrow \mathrm{BMI}^{* * *} \text { at } \\
6 \text { months }\end{array}$ & $\begin{array}{l}\downarrow \text { Fasting plasma } \\
\text { glucose }{ }^{*+} \text { but } \\
\text { returned to baseline } \\
\text { at } 12 \text { months } \\
\Delta 0.9 \text { mmol/L } \\
\downarrow 2 \text {-hr plasma } \\
\text { glucose }{ }^{* *} \text { but } \\
\text { returned to baseline } \\
\text { at } 12 \text { months } \\
\Delta 1.6 \text { mmol/L } \\
\downarrow \text { Fasting insulin **† } \\
\text { at } 18 \text { months } \\
\text { Cross-sectional } \\
\text { survey data between } \\
1993-1997 \text {, on wider } \\
\text { community: } \\
\downarrow \text { TC } \\
\text { (15-34 yrs) } \\
\downarrow \text { HC *** } \\
\text { (age-adjusted } \\
\text { prevalence at } \\
\text { baseline (31\%), } \\
2(21 \%) \text { and } 4 \text {-yrs } \\
\text { (15\%) } \\
\uparrow \text { Plasma } \\
\alpha \text {-tocopherol } \\
\uparrow \text { Plasma lutein and } \\
\text { zeaxanthin } \\
\uparrow \text { cryptoxanthin } \\
\uparrow \beta \text {-carotene }\end{array}$ & $\begin{array}{l}\downarrow \text { Total and } \\
\text { Saturated fat } \\
\uparrow \mathrm{FV} \\
\uparrow \mathrm{PA}\end{array}$ & $\begin{array}{l}\text { Unclear } \\
\text { (may still be } \\
\text { active in the } \\
\text { community) }\end{array}$ \\
\hline
\end{tabular}

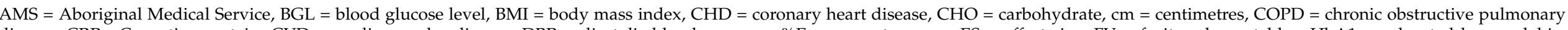

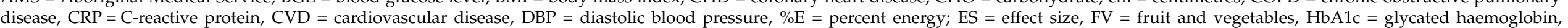

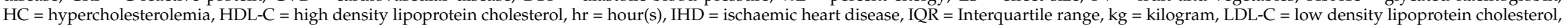

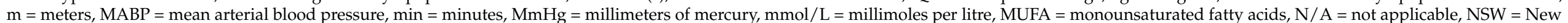

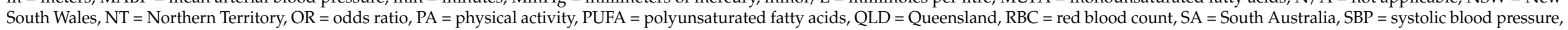

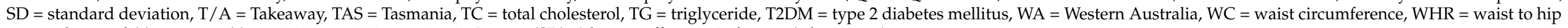
ratio, wk $=$ week(s), year $=\mathrm{yr}(\mathrm{s}),{ }^{*} \leq 0.05, * * \leq 0.01, * * * \leq 0.001,+$ mean (SD), $\beta$ beta-coefficient, $\Delta$ change, $\downarrow$ decrease, $\uparrow$ increase. 


\section{Results}

\subsection{Search Results}

The academic database search yielded a total number of 384 records using the search strategy on five electronic databases (Medline $=124$, Embase $=157$, Scopus $=81$, Informit Indigenous Collection $=21$, Informit Family and Society Collection $=1$ ). This was reduced to 235 after 149 duplicates were removed. A total of 235 records were then screened, with 225 records excluded. Ten reports were sought for full text retrieval and assessed for eligibility. Of these ten reports, a total of six reports representing five programs were identified through databases and included in this review.

A total of 430 records were identified via other methods, of which 22 full text reports were retrieved. Of these 22 reports, four were excluded as they were not primary research. A total of 18 reports, representing 16 programs, were identified through other methods and were included in this review. The flowchart in Figure 1 illustrates the process of the selection of sources of evidence.

\subsection{Program Selection and Characteristics}

A total of 24 reports were included in the review, representing 21 nutrition programs. Of these 21 programs, 12 were unpublished [32-44] and nine programs published in the scientific peer-reviewed literature [45-55]. Both unpublished programs (2000 to 2020) and peerreviewed published literature (1994 to 2014) spanned over a period of 20 years. Four programs were located in Queensland [32,39,43,46], Western Australia [34,48,49,51,54,55], Northern Territory [37,40,50,52], New South Wales [36,38,41,42] and one in Victoria [33], South Australia [45], Tasmania [47], Central Australia [53] and Australia-wide [44]. Nine programs were completed $[36,37,42,43,45-47,51-55]$, eight were active [32-35,38-40,44,48,49] and the status of four was unclear $[41,50,52,54,55]$. The participant size ranged from 10 to over 400 , but only $62 \%$ of the programs specified the total sample size. The program duration varied between one day and two years, while the time to follow-up ranged from eight weeks to eight years. One program included only female participants [36], and another included only male participants [42]. The delivery of programs was primarily in-person and group-based; however, two programs offered a telephone service $[39,44]$ and one provided an online component [39]. The education topics predominantly included healthy eating, weight loss and management, food budgeting, food label reading, and the benefits of exercise.

\subsection{Anthropometric Outcomes}

A total of 12 programs reported on anthropometric measurements [32,42,43,45-54]. Five programs produced significant reductions in BMI [45,48-50,52,54], four produced significant decreases in WC $[42,46,48-50]$, three reported significant decreases in weight $[42,45,50]$ and one reported significant reductions in WHR, fat mass and body fat percentage [50]. Effect sizes were used in one program to report reductions in weight, BMI and WC [47]. At an eight-year follow-up, one program reported significant increases to women's BMI and WC [53].

\subsection{Biochemical and/or Haematological Outcomes}

A total of 10 programs reported on biochemical and/or hematological outcomes [42-46,48,49,51-55]. Significant reduction in TC was the most common improvement in this category and was seen in four programs $[44,46,52,53]$, while a significant reduction in the prevalence of age-adjusted hypercholesterolemia (HC) was reported in one program [54,55] and a decrease in the odds (OR 0.29) of HC at eight years' follow-up for another [53]. Three programs produced significant reductions in HDL-C [42,46,53], while one program reported an increase, although its statistical significance is unclear [43]. One program reported significant improvements in targeted achievements in LDL-C [44]. A significant reduction in TG was reported for one program [46], while another program reported a significant increase in TG and dyslipidemia at the eight-year follow-up [53]. Three programs produced a significant decrease in SBP $[42,48,49,52]$ and DBP $[46,48,49,52]$. 
One program reported significant reductions in BGL [42], while another program saw significant decreases in two-hour plasma glucose, fasting glucose and fasting insulin; however, these reductions were not sustained $[54,55]$.

\subsection{Other Outcomes}

A total of 16 programs reported other outcomes [32-38,42-44,46-49,51-55]. Five programs reported improvements to diet quality [32,43,52-55], with the main improvement relating to increased consumption of fruits and vegetables. Three programs reported statistically significant improvements in fitness parameters and engagement in physical activity $[42,44,48,49]$, while improvements in physical activity were reported in nine others $[32,34-37,43,46,47,51,54]$. Other outcomes included improvements in participants knowledge and skills [32,34-36], self-esteem [34,35], well-being [43], fatigue levels and quality of life [47].

\subsection{Quality Appraisal}

The quality appraisal results are presented in Figure 2 with green representing (yes), blue (partially), orange (unclear) and red (no) for the responses to 126 questions. Green represented $35 \%$ of all the responses, followed by orange (28\%), blue (19\%) and red (18\%).

\begin{tabular}{|c|c|c|c|c|c|c|c|c|c|c|c|c|c|c|c|c|}
\hline Program name & $\begin{array}{l}\text { First author, } \\
\text { Year, } \\
\text { Reference, }\end{array}$ & Q1 & Q2 & Q3 & Q4 & Q5 & Q6 & Q7 & Q8 & Q9 & Q10 & Q11 & Q12 & Q13 & Q14 & $\begin{array}{l}\text { Overall } \\
\text { rating } \S\end{array}$ \\
\hline $\begin{array}{l}\text { The Aboriginal and Torres Strait } \\
\text { Islander Women's Fitness Program }\end{array}$ & Canuto, 2012 [45] & 0 & 0 & 0 & 0 & 0 & 0 & 0 & $\mathrm{O}$ & 8 & 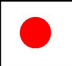 & 0 & 0 & 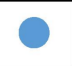 & O & Poor \\
\hline The Healthy Lifestyle Program & Chan, 2007 [46] & 0 & 0 & 0 & 0 & O & 0 & 0 & 0 & 0 & 0 & 0 & 0 & 0 & 0 & Poor \\
\hline $\begin{array}{l}\text { Cardiopulmonary rehabilitation and } \\
\text { secondary prevention program }\end{array}$ & Davey, 2014 [47] & 0 & 0 & 0 & 0 & 0 & 0 & 0 & 0 & 0 & O & 0 & $\mathrm{O}$ & 0 & O & Very good \\
\hline $\begin{array}{l}\text { Heart Health 'For Our People, By } \\
\text { Our People' }\end{array}$ & $\begin{array}{l}\text { Dimer, } 2013[48] \\
\text { Dimer, } 2012[49]\end{array}$ & 0 & 0 & 0 & $\mathrm{C}$ & 0 & 0 & 0 & O & C & 3 & 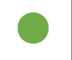 & O & 3 & 0 & Good \\
\hline GutBusters & Egger, 1999 [50] & 0 & O & O & 0 & O & 0 & 0 & O & O & 0 & 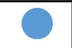 & O & O & O & Poor \\
\hline $\begin{array}{l}\text { Unity of First People of Australia } \\
\text { Diabetes Management Care }\end{array}$ & Gracey, 2006 [51] & O & 0 & 0 & 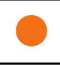 & O & 0 & 0 & 0 & ค & 0 & 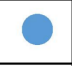 & O & 0 & 0 & Poor \\
\hline $\begin{array}{l}\text { The Minjilang Health and Nutrition } \\
\text { Project }\end{array}$ & Lee, 1994 [52] & 0 & 0 & O & 0 & 0 & 0 & 0 & 0 & 0 & 0 & 0 & 0 & 0 & 0 & Good \\
\hline Unnamed & $\begin{array}{l}\text { McDermott, } 2000 \\
{[53]}\end{array}$ & 0 & 0 & 0 & 0 & 0 & 0 & 0 & 0 & O & 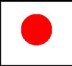 & 0 & O & O & O & Poor \\
\hline $\begin{array}{l}\text { The Looma Healthy Lifestyle } \\
\text { Project }\end{array}$ & $\begin{array}{l}\text { Rowley, } 2000[54] \\
\text { Rowley, } 2001[55]\end{array}$ & O & 0 & 0 & 0 & 0 & 0 & 0 & 0 & 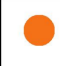 & ? & 0 & 0 & 0 & 0 & Good \\
\hline $\begin{array}{l}\text { Score } \\
\text { (ranking) }\end{array}$ & & $\begin{array}{l}14 \\
(6)\end{array}$ & $\begin{array}{l}23 \\
\text { (1) }\end{array}$ & $\begin{array}{l}12 \\
(7)\end{array}$ & $\begin{array}{l}8 \\
(9)\end{array}$ & $\begin{array}{l}21 \\
(3)\end{array}$ & $\begin{array}{l}12 \\
(7)\end{array}$ & $\begin{array}{l}14 \\
(6)\end{array}$ & $\begin{array}{l}16 \\
\text { (5) }\end{array}$ & \begin{tabular}{|l}
5 \\
$(10)$
\end{tabular} & $\begin{array}{l}11 \\
(8)\end{array}$ & $\begin{array}{l}16 \\
\text { (5) }\end{array}$ & $\begin{array}{l}22 \\
(2)\end{array}$ & $\begin{array}{l}23 \\
\text { (1) }\end{array}$ & $\begin{array}{l}18 \\
\text { (4) }\end{array}$ & \\
\hline
\end{tabular}

Key:

-Yes
-Unclear
-Partially
-No

Figure 2. The 2018 SAHMRI CREATE Aboriginal and Torres Strait Islander Quality Appraisal Tool results of peer-reviewed programs presented as an adapted traffic light plot. Q1 Did the research respond to a need or priority determined by the community?; Q2 Was community consultation and engagement appropriately inclusive?; Q3 Did the research have Aboriginal and Torres Strait Islander research leadership?; Q4 Did the research have Aboriginal and Torres Strait Islander governance?; Q5 Were local community protocols respected and followed?; Q6 Did the researchers negotiate agreements in regards to rights of access to Aboriginal and Torres Strait Islander peoples existing intellectual and cultural property?; Q7 Did the researchers negotiate agreements to protect Aboriginal and Torres Strait Islander peoples' ownership of intellectual and cultural property created through the research?; Q8 Did Aboriginal and Torres Strait Islander peoples and communities have control over the collection and management of research materials?; Q9 Was the research guided by an Indigenous research paradigm?; Q10 Does the research take a strengths-based approach, acknowledging and moving beyond practices that have harmed Aboriginal and Torres Strait peoples in the past?; Q11 Did the researchers plan to and translate the findings into sustainable changes in policy and/or practice?; Q12 Did the research benefit the participants and Aboriginal and Torres Strait Islander communities?; Q13 Did the research demonstrate capacity strengthening for Aboriginal and Torres Strait 
Islander individuals?; Q14 Did everyone involved in the research have opportunities to learn from each other? The symbol § represents the overall rating of peer-reviewed programs. A final rating of "very good" was assigned to papers that answered yes to over $75 \%$ of questions, "good" to those that answered yes to over $50 \%$ of questions, "fair" to those that answered yes to fewer than $50 \%$ of questions, and "poor" to those that answered yes to fewer than $25 \%$ of questions.

The program that performed best in terms of overall cultural appropriateness was by Davey et al. 2014 [47]. Three programs were rated as good [48,49,52,54,55] and the remaining five programs were rated as poor $[45,46,50,51,53]$. No trend was apparent regarding the date of programs and the ranking of cultural appropriateness, despite the most recent program receiving the highest ranking [47].

The three questions that received the highest score across all the programs were: Question 2, "Was the community consultation and engagement appropriately inclusive?"; Question 12, "Did the research benefit the participants and Aboriginal and Torres Strait Islander communities?"; and Question 13, "Did the research demonstrate capacity strengthening for Aboriginal and Torres Strait Islander individuals?". By contrast, the three lowestperforming questions were; Question 4, "Did the research have Aboriginal and Torres Strait Islander governance?"; Question 9, "Was the research guided by an Indigenous research paradigm?" and Question 10, "Does the research take a strengths-based approach, acknowledging and moving beyond practices that have harmed Aboriginal and Torres Strait Islander peoples in the past?".

\section{Discussion}

This scoping review is the first to map the evidence relating to the effectiveness of nutrition programs aiming to improve CVD outcomes in Aboriginal and Torres Strait Islander peoples and to appraise peer-reviewed interventions using a culturally appropriate Aboriginal and Torres Strait Islander QAT. Among the 21 programs identified, 12 programs were from unpublished research and nine from published research. Most programs reported improvements in measurable risk factors for CVD, including reduced BMI, WC, weight, blood pressure and improved lipid profiles. Other improvements included knowledge, skills, well-being and quality of life. It appears that a multifaceted education approach was consistently implemented. The predominant topics included healthy eating, weight loss and management, food budgeting, food label reading, and the benefits of exercise. Incorporating physical activity components was a common feature of many of the programs, which likely contributed to improved metabolic control. From the nine peer-reviewed publications that were critically appraised and evaluated using the QAT, four programs that were deemed more culturally appropriate were likely to be associated with program sustainability $[47-49,52,54,55]$ and may still be active within communities $[48,49,52,54,55]$.

Program evaluations help us to demonstrate impact and improve program design and implementation, as well as to drive support for funding. This scoping review found that not all programs had been evaluated and that of those that were evaluated, few were peer-reviewed. Peer-reviewed programs offered greater detail on anthropometric and biochemical and/or haematological measurements than non-peer-reviewed programs. Despite the limited number of peer-reviewed programs, the outcomes generally supported previous findings in that targeted cardiovascular programs incorporating both nutrition and physical activity are beneficial in reducing CVD risk and improving clinical outcomes [56,57]. Other qualitative and behavioural outcomes lacked consistency, were reported less frequently and were often based on anecdotal remarks. In the past, cultural components were not included as evaluable outcomes within nutrition programs for CVD [58]. A mixed method approach is often suitable for Aboriginal and Torres Strait Islander research, as qualitative feedback can provide valuable insight into the cultural relevance and acceptability of program components [59], factors of which can influence outcome measures. The most recent program in 2014 by Davey and colleagues rated best for overall cultural appropriateness [47] and was the only peer-reviewed program that reported improved quality of life and fatigue levels. 
Social determinants are estimated to contribute to over $30 \%$ of the health gap between Aboriginal and Torres Strait Islander peoples and non-Indigenous Australians [17]. Those with higher levels of education, income and food security have better health outcomes [60]. Interventions that provided multiple education components that address social determinants were identified to be effective in reducing measures across more than one anthropometric outcome. These educational components increased knowledge and awareness in the areas of healthy eating, weight loss and management, food budgeting, food label reading and the benefits of exercise. Consistent with a holistic health approach, additional education messaging was delivered on health literacy, wellbeing and stress management.

Another key program characteristic that appeared to correspond with improved outcome measures was a longer timeframe designated for interventions. A program that reduced measures in all the anthropometric outcomes of BMI, WC, weight, WHR and fat reduction was delivered over 12 months [50]. Similarly, the two interventions that performed well at reducing measures within multiple biochemical/haematological outcomes were delivered across communities over a longer time-frame of 2 years [46] and 8 years [53]. Emerging evidence suggests that long-term changes in diet quality correlate with a lower risk of CVD and mortality [61]. Interestingly, the three programs that performed well across clinical measures scored 'poor' in the quality appraisal $[46,50,53]$.

Scholarship in the areas of Indigenous epistemology, ontology and axiology has led to an understanding of Aboriginal and Torres Strait Islander ways of knowing as fluid, meaning that it can evolve and are influenced by situational contexts, such as the political and social environment [30,31]. The timeline towards healing and reconciliation, as seen through the social justice report and the Close the Gap and Closing the Gap campaigns have established a discourse amongst academics and researchers to promote Indigenous paradigms in research $[14,15,30]$. This privileging of Aboriginal and Torres Strait Islander knowledge has led to the development of quality appraisal tools to assess programs not only through a scientific lens but also through an Aboriginal and Torres Strait Islander cultural lens [24].

This review retrospectively assessed the cultural appropriateness of programs and highlighted that of the programs evaluated and peer-reviewed, less than half of the programs answered 'yes' to $50 \%$ or more of the quality appraisal questions. As evidenced, most programs performed well at community engagement and capacity strengthening but they lacked the inclusion of Indigenous research paradigms, governance and taking a strengths-based approach. The lack of inclusion of cultural components within the program should be interpreted with consideration that they were reported between 1994 and 2014 and the Aboriginal and Torres Strait Islander QAT used herein was developed in 2018 and published in 2020 [24]. It is possible that the 'poor' QAT score assigned to programs is reflective of the scholarship and understanding in Aboriginal and Torres Strait Islander research at that time. Importantly, this indicates a gap in research and highlights a need for contemporary nutrition programs aimed at improving cardiovascular health outcomes to include additional key cultural components.

An extensive and wide search was a strength of this review, scoping all the available published and unpublished literature. Further, to the author's knowledge, this review was the first to appropriately apply a quality appraisal tool from an Indigenous perspective to ascertain the cultural appropriateness of nutrition programs targeting CVD. A limitation to the review was that only a small number of programs was evaluated, which resulted in limited application of the QAT. Furthermore, this scoping review retrospectively assessed the cultural appropriateness of programs as most peer-reviewed studies retrieved were published between 1994 to 2014, before the development of the QAT in 2018. It is important that the value and relevance of the previous research and programs presented in this review are acknowledged. The findings are not a reflection of overall poor cultural appropriateness, but rather a reflection of the advancement of knowledge in the areas of Indigenous epistemology, ontology, axiology and research methodologies. 


\section{Conclusions}

Future nutrition programs aiming to improve cardiovascular health outcomes should be multifaceted, incorporating both nutrition and physical activity. Most programs identified in this review undertook community consultation, engagement and capacity strengthening, but improvements in key areas of Indigenous paradigm, governance and strengthsbased approaches are required. Future research should focus on sustainability within communities through effective co-design with Aboriginal and Torres Strait Islander peoples and communities.

Supplementary Materials: The following are available online at https:/ /www.mdpi.com/article/10 $.3390 /$ nu13114084/s1. Table S1: Medline Search Strategy.

Author Contributions: Conceptualization, B.P., A.D. and M.A.-F.; methodology, B.P., A.D., C.B., H.M., J.C. and M.A.-F.; formal analysis, B.P., A.D., C.B. and H.M.; J.C. writing-original draft preparation, B.P., A.D., C.B. and H.M.; writing-review and editing, J.C. and M.A.-F.; supervision, B.P., A.D., J.C. and M.A.-F. All authors have read and agreed to the published version of the manuscript.

Funding: This research received no external funding.

Acknowledgments: We acknowledge the contribution of the University of Technology Sydney, School of Public Health, for funding publication costs. We acknowledge the contribution of Genus Passi for highlighting a community need for culturally appropriate nutrition interventions amongst Aboriginal and Torres Strait Islander peoples in Far North Queensland.

Conflicts of Interest: The authors declare they have no conflict of interest.

\section{References}

1. Australian Human Rights Commission. Bringing Them Home, Report of the National Inquiry into the Separation of Aboriginal and Torres Strait Islander Children from Their Families. 1997. Available online: https://humanrights.gov.au/sites/default/files/ content/pdf/social_justice/bringing_them_home_report.pdf (accessed on 29 September 2021).

2. Ferguson, M.; Brown, C.; Georga, C.; Miles, E.; Wilson, A.; Brimblecombe, J. Traditional food availability and consumption in remote Aboriginal communities in the Northern Territory, Australia. Aust. N. Z. J. Public Health 2017, 413, 294-298. [CrossRef]

3. Australian Indigenous HealthInfoNet. Summary of Nutrition among Aboriginal and Torres Strait Islander People. 2020. Available online: https: / / apo.org.au/sites/default/files/resource-files/2020-05/apo-nid305130.pdf (accessed on 29 September 2021).

4. Brimblecombe, J.; Liddle, R.; O'Dea, K. Use of point-of-sale data to assess food and nutrient quality in remote stores. Public Health Nutr. 2013, 167, 1159-1167. [CrossRef] [PubMed]

5. Brimblecombe, J.K.; Ferguson, M.M.; Liberato, S.C.; O’Dea, K. Characteristics of the community-level diet of Aboriginal people in remote northern Australia. Med. J. Aust. 2013, 1987, 380-384. [CrossRef]

6. Lee, A.J.; O’Dea, K.; Mathews, J.D. Apparent dietary intake in remote Aboriginal communities. Aust. J. Public Health 1994, 182, 190-197. [CrossRef] [PubMed]

7. Brandhorst, S.; Longo, V.D. Dietary Restrictions and Nutrition in the Prevention and Treatment of Cardiovascular Disease. Circ. Res. 2019, 1246, 952-965. [CrossRef]

8. Australian Institute of Health and Welfare. Cardiovascular Disease. Available online: https://www.aihw.gov.au/reports/heartstroke-vascular-diseases / cardiovascular-health-compendium (accessed on 14 July 2021).

9. Australian Indigenous HealthInfoNet. Summary of Aboriginal and Torres Strait Islander Health Status 2019 (Overview). 2020. Available online: https:/ / healthinfonet.ecu.edu.au/healthinfonet/getContent.php?linkid $=643680 \&$ title=Summary + of + Aboriginal+and+Torres+Strait+Islander+health+status+2019\&contentid=40279_1 (accessed on 24 September 2021).

10. World Health Organization. Cardiovascular Diseases. Available online: https:/ /www.who.int/health-topics/cardiovasculardiseases/\#tab=tab_1 (accessed on 14 July 2021).

11. Australian Institute of Health and Welfare. Trends in Cardiovascular Deaths. 2017. Available online: https://www.aihw.gov.au/ getmedia/2ba74f7f-d812-4539-a006-ca39b34d8120/aihw-21213.pdf (accessed on 24 September 2021).

12. Australian Institute of Health and Welfare. Coronary Heart Disease. 2020. Available online: https://www.aihw.gov.au/reports/ australias-health/coronary-heart-disease (accessed on 24 September 2021).

13. Australian Institute of Health and Welfare. Australian Burden of Disease Study: Impact and Causes of Illness and Death in Aboriginal and Torres Strait Islander People 2011-Summary Report. 2016. Available online: https://www.aihw.gov.au/reports/ burden-of-disease/illness-death-indigenous-australians-summary/contents/table-of-contents (accessed on 24 September 2021).

14. Australian Human Rights Commission. Social Justice Report 2005: Home. 2005. Available online: https://humanrights.gov.au/ourwork/aboriginal-and-torres-strait-islander-social-justice/publications/social-justice-report-5 (accessed on 29 September 2021).

15. Australian Department of the Prime Minister and Cabinet. Closing the Gap Report 2020. Available online: https://ctgreport.niaa. gov.au/sites/default/files/pdf/closing-the-gap-report-2020.pdf (accessed on 14 July 2021). 
16. Wilson, S. Progressing Toward an Indigenous Research Paradigm in Canada and Australia. Can. J. Nativ. Educ. 2003, $272,161$.

17. Australian Institute of Health and Welfare. Social Determinants and Indigenous Health; AIHW: Canberra, Australia, 2020.

18. Hart, A.C.; Rosewarne, E.; Spencer, W.; McCausland, R.; Leslie, G.; Shanthosh, J.; Corby, C.; Bennett-Brook, K.; Webster, J. Indigenous Community-Led Programs to Address Food and Water Security: Protocol for a Systematic Review. Int. J. Environ. Res. Public Health 2021, 18, 6366. [CrossRef] [PubMed]

19. Australian Institute of Health and Welfare. What Works? A Review of Actions Addressing the Social and Economic Determinants of Indigenous Health. Available online: https://www.aihw.gov.au/reports/indigenous-australians/what-works-a-reviewof-actions-addressing-the-social-and-economic-determinants-of-indigenous-health/contents/table-of-contents (accessed on 14 July 2021).

20. Australian Government Productivity Commission. Better Indigenous Policies: The Role of Evaluation. Available online: https:/ / www.pc.gov.au/research/supporting/better-indigenous-policies (accessed on 14 July 2021).

21. Capwell, E.M.; Butterfoss, F.; Francisco, V.T. Why Evaluate? Health Promot. Pract. 2000, 11, 15-20. [CrossRef]

22. Demaio, A.; Drysdale, M.; de Courten, M. Appropriate health promotion for Australian Aboriginal and Torres Strait Islander communities: Crucial for closing the gap. Glob. Health Promot. 2012, 192, 58-62. [CrossRef] [PubMed]

23. Australian Government Productivity Commission. Indigenous Evaluation Strategy. Available online: https://www.pc.gov.au/ inquiries/completed/indigenous-evaluation\#report (accessed on 14 July 2021).

24. Harfield, S.; Pearson, O.; Morey, K.; Kite, E.; Canuto, K.; Glover, K.; Gomersall, J.S.; Carter, D.; Davy, C.; Aromataris, E.; et al. Assessing the quality of health research from an Indigenous perspective: The Aboriginal and Torres Strait Islander quality appraisal tool. BMC Med. Res. Methodol. 2020, 20, 79. [CrossRef]

25. Munn, Z.; Peters, M.D.J.; Stern, C.; Tufanaru, C.; McArthur, A.; Aromataris, E. Systematic review or scoping review? Guidance for authors when choosing between a systematic or scoping review approach. BMC Med. Res. Methodol. 2018, 18, 143. [CrossRef] [PubMed]

26. Arksey, H.; O'Malley, L. Scoping studies: Towards a methodological framework. Int. J. Soc. Res. Methodol. 2005, 81, 19-32. [CrossRef]

27. Peters, M.D.J.; Marnie, C.; Tricco, A.C.; Pollock, D.; Munn, Z.; Alexander, L.; McInerney, P.; Godfrey, C.M.; Khalil, H. Updated methodological guidance for the conduct of scoping reviews. JBI Evid. Synth. 2020, 1810, 2119-2126. [CrossRef]

28. Harfield, S.; Pearson, O.; Morey, K.; Kite, E.; Glover, K.; Canuto, K.; Streak, G.J.; Carter, D.; Davy, C.; Aromataris, E.; et al. The Aboriginal and Torres Strait Islander Quality Appraisal Tool: Companion Document. Adelaide, Australia: South Australian Health and Medical Research Institute. 2018. Available online: https://create.sahmri.org/wp-content/uploads/2020/04/ Aboriginal-and-Torres-Strait-Islander-QAT-Companion-Document.pdf (accessed on 5 August 2021).

29. Laycock, A.; Walker, D.; Harrison, N.; Brands, J. Supporting Indigenous Researchers: A Practical Guide for Supervisors, Cooperative Research Centre for Aboriginal Health, Darwin. 2009. Available online: https://www.lowitja.org.au/content/ Document/Lowitja-Publishing/supervisors_guide1_0.pdf (accessed on 5 August 2021).

30. Martin, K.; Mirraboopa, B. Ways of knowing, being and doing: A theoretical framework and methods for indigenous and indigenist research. J. Aust. Stud. 2003, 2776, 203-214. [CrossRef]

31. Smith, L.T. Decolonizing Methodologies: Research and Indigenous Peoples, 3rd ed.; Zed Books: London, UK, 2021.

32. Queensland Health. 2005 Evaluation of the Healthy Weight Program; Queensland Government: Brisbane, Australia, 2005.

33. Life! Aboriginal Road to Good Health. 2016. Available online: https://www.lifeprogram.org.au/about-the-life-program/aboutthe-program/aboriginal-road-to-good-health (accessed on 15 July 2021).

34. Dimer, L.; Shilton, T.; Hayes, A.; Hayes, S.; Heard, M.; Turangi, G.; Hamilton, S. Listen to the Voice of the People: Culturally specific consultation and innovation in establishing an Aboriginal health program in the west Pilbara. In Proceedings of the 14th National Rural Health Conference; Heart Foundation WA: Cairns, Australia, 2017.

35. Public Health Advocacy Institute of Western Australia. The West Australian Indigenous Storybook. Fremantle: Public Health Advocacy Institute of Western Australia. 2018. Available online: https://www.phaiwa.org.au/wp-content/uploads/2019/03/ TheWAIndigenousStorybook_9thEd_E.pdf (accessed on 15 July 2021).

36. Zask, A.; Rodgers, L.; Dietrich, U. Healing Program 2003-2004 Evaluation Report; NSW Helath Department, Ed.; North Coast Area Health Service: Lismore, Australia, 2007.

37. Alexander, S. Cooking Healthy and Physical Activity (CHAPA) Project. Chronicle 2009, 121, 15.

38. Heart Foundation. Steps to Running Your Own Koori Cook Off. 2017. Available online: http://www.gdcbj.com/images/ uploads/main/Heart_Foundation_-_Koori_Cook_Off_Toolkit.pdf (accessed on 15 July 2021).

39. Hamill, L.; Clark, K. My Heath for Life: An Innovative, Evidence-Based Preventative Health Program for Tackling Chronic Disease in Queensland. In Program Design and Delivery Overview; Queensland Government, Ed.; Diabetes Queensland: Brisbane, Australia, 2016.

40. Wurli-Wurlinjang Health Service. Gudbinji Chronic Conditions Care. Available online: https://www.wurli.org.au/clinicalservices / gudbinji-chronic-conditions-program/ (accessed on 15 July 2021).

41. Green, M.; Dangerfield, F. “Urimbirra Geen”-To Take Care of Your Heart. Aborig. Isl. Health Work. J. 2000, $24,3-5$.

42. Biles, B. 'Strong Men': Aboriginal Community Development of a Cardiovascular Exercise and Health Education Program. Ph.D Thesis, Charles Sturt University, Bathurst, Australia, 2020.

43. Heath, D.; Longstreet, D.; Malouf, P.; Hussey, L.; Elston, J.; Panaretto, D. “Walkabout Together" A lifestyle intervention program developed for Townsville's overweight Indigenous people. Touch PHAA Newsl. 2006, 23, 17-18. 
44. Ski, C.; Vale, M.; Thompson, D.; Jelinek, M.; Scott, I.; Le Grande, M. The Coaching Patients on Achieving Cardiovascular Health $(\mathrm{COACH})$ Programme: Reducing the Treatment Gap Between Indigenous and Non-Indigenous Australians. Heart Lung Circ. 2017, 26, S336. [CrossRef]

45. Canuto, K.; Cargo, M.; Li, M.; D’Onise, K.; Esterman, A.; McDermott, R. Pragmatic randomised trial of a 12-week exercise and nutrition program for Aboriginal and Torres Strait Islander women: Clinical results immediate post and 3 months follow-up. BMC Public Health 2012, 121, 933. [CrossRef]

46. Chan, L.C.K.; Ware, R.; Kesting, J.; Marczak, M.; Good, D.; Shaw, J.T.E. Short term efficacy of a lifestyle intervention programme on cardiovascular health outcome in overweight Indigenous Australians with and without type 2 diabetes mellitus: The healthy lifestyle programme (HELP). Diabetes Res. Clin. Pract. 2007, 751, 65-71. [CrossRef]

47. Davey, M.; Moore, W.; Walters, J. Tasmanian Aborigines step up to health: Evaluation of a cardiopulmonary rehabilitation and secondary prevention program. BMC Health Serv. Res. 2014, 141, 349. [CrossRef]

48. Dimer, L.; Dowling, T.; Jones, J.; Cheetham, C.; Thomas, T.; Smith, J.; McManus, A.; Maiorana, A.J. Build it and they will come: Outcomes from a successful cardiac rehabilitation program at an Aboriginal Medical Service. Aust. Health Rev. 2013, $371,79-82$. [CrossRef] [PubMed]

49. Dimer, L.; Jones, J.; Dowling, T.; Cheetham, C.; Maiorana, A.; Smith, J. Heart Health for Our People by Our People: A Culturally Appropriate WA CR Program. Heart Lung Circ. 2012, 2110, 651-652. [CrossRef]

50. Egger, G.; Fisher, G.; Piers, S.; Bedford, K.; Morseau, G.; Sabasio, S.; Taipim, B.; Bani, G.; Assan, M.; Mills, P. Abdominal obesity reduction in indigenous men. Int. J. Obes. Relat. Metab. Disord. 1999, 236, 564-569. [CrossRef] [PubMed]

51. Gracey, M.; Bridge, E.; Martin, D.; Jones, T.; Spargo, R.M.; Shephard, M.; Davis, E.A. An Aboriginal-driven program to prevent, control and manage nutrition-related "lifestyle" diseases including diabetes. Asia Pac. J. Clin. Nutr. 2006, 152, $178-188$.

52. Lee, A.J.; Bailey, A.P.; Yarmirr, D.; O’Dea, K.; Mathews, J.D. Survival tucker: Improved diet and health indicators in an aboriginal community. Aust. J. Public Health 1994, 183, 277-285. [CrossRef]

53. McDermott, R.; Rowley, K.G.; Lee, A.J.; Knight, S.; O'Dea, K. Increase in prevalence of obesity and diabetes and decrease in plasma cholesterol in a central Australian aboriginal community. Med. J. Aust. 2000, 17210, 480-484. [CrossRef]

54. Rowley, K.G.; Daniel, M.; Skinner, K.; Skinner, M.; White, G.A.; O’Dea, K. Effectiveness of a community-directed “healthy lifestyle" program in a remote Australian aboriginal community. Aust. N. Z. J. Public Health 2000, 242, 136-144. [CrossRef]

55. Rowley, K.G.; Su, Q.; Cincotta, M.; Skinner, M.; Skinner, K.; Pindan, B.; White, G.A.; O’Dea, K. Improvements in circulating cholesterol, antioxidants, and homocysteine after dietary intervention in an Australian Aboriginal community. Am. J. Clin. Nutr. 2001, 744, 442-448. [CrossRef]

56. Tsai, M.-C.; Lee, C.-C.; Liu, S.-C.; Tseng, P.-J.; Chien, K.-L. Combined healthy lifestyle factors are more beneficial in reducing cardiovascular disease in younger adults: A meta-analysis of prospective cohort studies. Sci. Rep. 2020, 10, 1-10. [CrossRef]

57. Hwang, W.J.; Kang, S.J. Interventions to Reduce the Risk of Cardiovascular Disease among Workers: A Systematic Review and Meta-Analysis. Int. J. Environ. Res. Public Health 2020, 177, 2267. [CrossRef] [PubMed]

58. Mbuzi, V.; Fulbrook, P.; Jessup, M. Effectiveness of programs to promote cardiovascular health of Indigenous Australians: A systematic review. Int. J. Equity Health 2018, 17, 1-17. [CrossRef]

59. Moore, G.F.; Audrey, S.; Barker, M.; Bond, L.; Bonell, C.; Hardeman, W.; Moore, L.; O'Cathain, A.; Tinati, T.; Wight, D.; et al. Process evaluation of complex interventions: Medical Research Council guidance. BMJ Br. Med. J. 2015, 350, h1258. [CrossRef] [PubMed]

60. Marmot, M.P.; Friel, S.P.; Bell, R.P.; Houweling, T.A.J.P.; Taylor, S.P. Closing the gap in a generation: Health equity through action on the social determinants of health. Lancet 2008, 3729650, 1661-1669. [CrossRef]

61. Livingstone, K.M.; Abbott, G.; Bowe, S.J.; Ward, J.; Milte, C.; McNaughton, S.A. Diet quality indices, genetic risk and risk of cardiovascular disease and mortality: A longitudinal analysis of 77004 UK Biobank participants. BMJ Open 2021, 114, e045362. [CrossRef] 